\title{
Trasplantation of Exosomes Derived From CD90 Positive Fibroblasts Reduce Apoptosis of Cardiomyocytes in Mice After Acute Myocardial Infarction
}

Qi Li

First Affiliated Hospital of Jinzhou Medical University

\section{Xiaolei Shen}

First Affiliated Hospital of Jinzhou Medical University

\section{Shan Wen}

First Affiliated Hospital of Jinzhou Medical University

\section{Yuanlong Li}

First Affiliated Hospital of Jinzhou Medical University

Dong-Mei Xie ( $\square$ xiedmys@foxmail.com)

Sun Yat-Sen University https://orcid.org/0000-0001-8222-6369

\section{Research}

Keywords: Myocardial infarction, CD90 positive fibroblasts, exosome, apoptosis, PI3K/AKT signaling pathway.

Posted Date: March 11th, 2021

DOI: https://doi.org/10.21203/rs.3.rs-266979/v1

License: (c) (i) This work is licensed under a Creative Commons Attribution 4.0 International License. Read Full License 
Title page:

Transplantation of exosomes derived from CD90 positive fibroblasts reduce apoptosis of cardiomyocytes in mice after acute myocardial infarction

Qi Li ${ }^{1 *}$, Xiaolei Shen ${ }^{1}$, Shan Wen ${ }^{1}$, Yuanlong $\mathrm{Li}^{1 \#}$, Dongmei Xie ${ }^{1 \#}$

${ }^{1}$ The First Affiliated Hospital of Jinzhou Medical University, Liaoning, China.

\# correspondence to: Yuanlong Li, The First Affiliated Hospital of Jinzhou Medical University, Liaoning, China, liyuanlong2015@foxmail.com; Dongmei Xie, The First Affiliated Hospital of Jinzhou Medical University, Liaoning, China, xiedmys@foxmail.com.

All authors declare no financial conflict of interest.

keywords: Myocardial infarction, CD90 positive fibroblasts, exosome, apoptosis, PI3K/AKT signaling pathway.

\begin{abstract}
Myocardial infarction (MI) leads to cardiomyocyte death resulting in cardiac dysfunction. However, the molecular mechanism is still poorly understood. Cardiac fibroblasts can be marked by CD90, a N-glycosylated glycophosphatidylinositol
\end{abstract}


anchored conserved cell surface protein, and the CD90 positive cardiac fibroblasts can release exosome against ischemic damage. Reduce apoptosis can promote the recovery of myocardial function after ischemic heart. Interestingly, exosome from CD90 positive cardiac fibroblasts can decrease the level of apoptosis after MI in vivo. However, the ability of CD90 positive cardiac fibroblasts exosome to reduce apoptosis, and the potential effects on the recovery of cardiomyocytes in heart after MI remain to be clarified. In this study, we found that CD90 positive cardiac fibroblasts exosome treated induced myocardial function recovery in a mouse MI model. Indeed, CD90 positive cardiac fibroblasts exosome increased phosphatidylinositol 3-kinase (PI3K) and protein kinase B (AKT) after MI. In addition, CD90 positive cardiac fibroblasts exosome decreased TUNEL in cardiomyocytes after MI. Furthermore, PI3K was inhibited by PI3K inhibitor LY294002, while hypoxia-stimulated primary cardiomyocytes were treated with CD90 positive cardiac fibroblasts exosome. Collectively, our results suggest that CD90 positive cardiac fibroblasts exosome can improve the recovery of cardiac function by decreasing apoptosis after MI in mice, likely via the PI3K/AKT signaling pathway.

keywords: Myocardial infarction, CD90 positive fibroblasts, exosome, apoptosis, PI3K/AKT signaling pathway.

\section{Introduction}


As always, ischemic heart disease is one of the most widely and threatening killers to humans, especially the elderly (1). Until now, numerous dedication was made to reduce the poor outcomes in patients with heart disease, and strategies were included during the process of precautions, diagnostics, and treatments $(2,3)$. Considering the regenerative capacity of adult cardiomyocytes was extremely low and it was insufficient to meet the functional and structural requirements after the myocardium was injured, an increasing number of researches were focused on the preservation of cardiomyocytes through cellular transplantation with or without cytokines supplement $(4,5)$. However, the number of exogenous cells that migrated and retained in the myocardium was not optimistic, because many cells were located in the lungs or spleens(6). Currently, researches indicated that cells from the auto tissues of the exact organs quickly and accurately facilitated organ repair after they were injured(7-9).

In hearts, cardiac fibroblasts occupied a moderate count and were involved in the synthesis of extracellular membrane during the physiological and pathophysiological processes(10). Also, cardiac fibroblasts moderately resisted ischemic damage, and participated in the regulation of prognosis in heart disease patients(11-13). CD90 is a N-glycosylated glycophosphatidylinositol anchored conserved cell surface protein through a single V-like immunoglobulin domain(14). Recently, CD90 was used to mark subpopulation of mesenchymal stem cells, induced pluripotent stem cells, or fibroblasts, and exhibited promising therapeutic efficiency(15). Yuqiao Chang et al. have proved that cardiac derived CD90 positive fibroblasts effectively reduced fibrosis and 
preserved the heart function in the rats after acute myocardial infarction(16). However, the underlying mechanism was not clear. Meanwhile, transplantation of numerous fibroblasts into the hearts may not be safe for patients in the clinic for the reason of the arrhythmia complications. Therefore, cell-free therapy is more acceptable.

Exosome, lipid enclosed extracellular vesicle, were produced in the endosomal compartment of cells in vitro or in vivo(17). They possessed specialized functions during physiological and pathological processes and played an essential role in the diagnosis or therapy of diseases. Consistently, a growing interest of exosome researches was focus on the cardiac field(18). The exosomes secreted from stem cells and human induced pluripotent stem cells were used to treat myocardial infarction disease(19). As the therapeutic potential of exosomes were profound, more researches should be implemented to verify the cellular sources associated with secretion of the exosome. Interestingly, the secreted factors from cardiac fibroblasts were valid to protect cardiomyocytes from ischemia injury $(20,21)$. We believed that the exosome from CD90 positive cardiac fibroblasts would contribute to the repair of the heart after acute myocardial infarction.

In this study, exosome was isolated from the primary CD90 positive cardiac fibroblasts, and their characteristics were verified by western blots. To elucidate the function of exosome to mice with acute myocardial infarction injury, heart functions were assessed by echocardiography and pathological changes were evaluated by 
immunohistochemically staining. In addition, the underlying mechanisms were analyzed by immunofluorescent staining and qPCR (Real-time Quantitative PCR) in vivo to address the biological process.

\section{Materials and Methods}

\section{Experimental grouping}

This experiment is divided into three parts: exosomes extraction and identification, exosomes in vivo validation experiments, exosomes in vitro validation experiments. The first part of the experiment was divided into two groups: EXO (exosomes form fibroblasts), Cell (fibroblasts only); The second part of the experiment was divided into three groups: sham (only open the chest without damaging the myocardium), injury (myocardial infarction model), EXO (exosomes from fibroblasts in the treatment of myocardial infarction); The last part of the experiment was divided into two groups: injury (cardiomyocytes under hypoxia condition), EXO (exosomes treatment group).

\section{Isolation and culture of fibroblasts and cardiomyocytes}

Neonatal C57BL/6 mice (1-2 days old) were used for cellular isolation following the instruction from the lab of Anthony O. Gramolini(22). Ventricles were collected and minced in HBSS containing $1 \% \mathrm{~m} / \mathrm{v}$ collagenase II (Gibco, USA) and $1 \% \mathrm{v} / \mathrm{v}$ pancreatin (Sigma, USA) for 2 hours at $37^{\circ} \mathrm{C}$ temperatures on a water bath shaker. The 
digested tissues were gently dissolved by Pasteur pipette and centrifuged at $1000 \mathrm{~g}$ for 10 minutes in $4^{\circ} \mathrm{C}$ temperatures. The cell sediments were re-suspended in DMEM/F12 (Dulbecco's Modified Eagle Media: Nutrient Mixture F-12, Hyclone) medium supplemented with 10\% FBS (fatal bovine serum, Sigma) and 1\% streptomycin penicillin (Sigma), and plated on $100 \mathrm{~mm}$ tissue culture dishes, incubated at $37{ }^{\circ} \mathrm{C}$ for 60 minutes. The non-adherent cardiomyocytes were transferred to $24-w e l l$ plates, and maintained in DMED/F12 with 10\% FBS, 1\% streptomycin penicillin. These cells are regarded as cardiomyocytes and are used for the detection of cardiomyocytes. The adherent cells were regarded as fibroblasts and maintained in DMEM/F12 medium supplemented with $10 \%$ FBS, $1 \%$ streptomycin penicillin and nonessential amino acids.

Flow cytometry assays were performed to sort CD90 positive fibroblasts. Fibroblasts were digested when the cell density reached to $95 \%$. After the count, cells were centrifuged and re-suspended in FACS buffer containing CD90 monoclonal antibody (Invitrogen, $5 \mu \mathrm{L}$ antibodies per $1 \times 10^{5}$ cells). After incubation for 30 minutes at $4{ }^{\circ} \mathrm{C}$, cells were washed by FACS buffer for two times, and transferred to a polystyrene round bottom tube (BD, USA) for FACS. The CD90 positive fibroblasts were collected and cultured in medium described above, and the cells used for isolating exosomes were propagated less than twice.

\section{Immunohistochemically staining}


For cultured cells, cells were washed by PBS (phosphate buffered saline) for 2 times, and fixed with $4 \%$ paraformaldehyde for 30 minutes, followed by permeabilization in $0.2 \%$ triton for 15 minutes. After blocking in 5\% BSA (Bovine Serum Albumin) for 1 hour, cells were treated with antibodies of FSP1(Abcam, USA), CD90(Abcam), $\alpha$ actinin (Abcam) at $4{ }^{\circ} \mathrm{C}$ overnight. Sequentially, cells were exposed to the appropriate secondary antibodies with or without co-incubated with Tunel (Thermofic, USA) for 60 minutes at $4{ }^{\circ} \mathrm{C}$. Nucleus was stained with DAPI (2-(4-Amidinophenyl)-6indolecarbamidine dihydrochloride). For tissues, hearts were collected and sliced in paraffin sections for a thickness of $6 \mu \mathrm{m}$. The paraffin sections were stained with hematoxlyin and eosin (H\&E) solution, or Masson staining solution directed by the instructions. Images were photographed under Olympus confocal laser scanning microscope or light microscopy.

\section{Isolation and characterization of exosome}

The culture media of CD 90 positive fibroblasts was collected and centrifuged at 3000 rpm (20 min), then the supernatant can be collected. Further, the supernatant was centrifuged at $100000 \mathrm{rpm}(1.5 \mathrm{~h})$ to concentrate the exosome. The characterizations of the exosomes were determined using a transmission electron microscope (TEM) (Hitachi, Tokyo, Japan, HT770). Particle size and surface charge were texted by dynamic light scattering (DLS) (Zetasizer Nano ZS; Malvern Instruments, Malvern, 
UK). After that, we tested the markers of exosome including CD63, CD81, TSG101 and GRP94 using Western blot.

\section{Western blot assay}

In Western blot, we used RIPA (Radio-Immunoprecipitation Assay) and PMSF (Phenylmethanesulfonyl fluoride, Sigma, USA) to get the protein of the cells and tissue from each group. After electrophoresis, the membranes were incubated with the primary antibodies, including anti-CD63 antibody (1:1000, abcam, USA, ab59479), anti-CD81 antibody (1:1000, abcam, USA, ab219209), anti-CD90 antibody (1:1000, abcam, USA, ab225), anti-TSG101 (1:500, abcam, USA, ab125011), anti-GRP94 (1:500, abcam, USA, ab2791), anti-Cleaved Caspase 3 (1:800, abcam, USA, ab2302), anti-BAX (1:800, abcam, USA, ab77566), anti-BCL-2 (1:800, abcam, USA, ab196495), anti-BAX (1:1000, abcam, USA, ab181602). Next, the membranes were washed by PBS, and incubated by the following secondary antibodies: Affinpure horseradish peroxidase (HRP) -conjugated goat anti-rabbit $\lg$ (1:5000, Haoranbio, Shanghai, China, HSA0003) or anti-mouse $\operatorname{lgG}($ HSA0001). Finally, the membranes were washed by PBS and the proteins were visualized by ECL solution. The data was quantified by Image $2 \mathrm{X}$ software.

\section{Acute myocardial infarction model}


C57BL/6 mice ( 8 weeks old age) were anesthetized and conducted for skin preparation. Then, they were intubated and connected to a small animal ventilator. Sequentially, thoracotomy was performed between the fourth and fifth intercostal at the left side to expose the heart. After removed the pericardium, the left anterior descending coronary artery was ligated by 9-0 noninvasive silk thread. After ischemia was confirmed, exosomes $\left(2 \times 10^{6} / \mathrm{mL}, 25 \mu \mathrm{L}\right.$ per mouse $)$ or saline were injected into the myocardium near the pale area. Finally, the thoracic cavity was sutured, and electrocardiogram was performed to verify infarction. For the sham-operation groups, the left anterior descending coronary artery was not ligated. During raising, the survival rate of the sham-operation, injury and exosome treated mice were recorded. At 4 weeks after surgery, mice were sacrificed for the experiments, and heart/body weight was recorded.

\section{Cardiac function assessment by echocardiography}

Four weeks after surgery, cardiac function of mice was assessed by ultrasonic cardiogram using a high-resolution small animal ultrasound imaging system (Vevo 2100, VisualSonics, Canada). And the following parameters were recorded: left ventricular ejection fraction (LVEF), left ventricular fractional shortening (LVFS), left ventricular end systolic diameter (LVESD), left ventricular end-diastolic diameter (LVEDD).

\section{TTC staining}


In order to assess infarct size, Triphenylterazolium chloride (TTC) staining was performed. Hearts were collected at 4 weeks after surgery and cut into $1 \mathrm{~mm}$ thick slice perpendicular to the long axis. All slices were incubated in $1 \%$ TTC at $37{ }^{\circ} \mathrm{C}$ for 15 minutes. The images were obtained by mobile phone and analyzed by Image $\mathrm{J}$ software. Infarct ratios were presented as a percentage of white area to the total ventricular area.

\section{MTT assays}

To measure the suitable concentration of exosome treatment after injury in vitro, 3(4,5-Dimethylthiazol-2-yl)-2,5-diphenyltetrazolium bromide (MTT) assays were used to evaluate cardiomyocytes viability after hypoxia. Cardiomyocytes were treated with exosome $\left(0,5 \times 10^{2}, 1 \times 10^{3}, 5 \times 10^{3}, 1 \times 10^{4}, 5 \times 10^{4}\right.$ and $\left.1 \times 10^{5}\right)$ and hypoxia $\left(2 \% \mathrm{O}_{2}\right)$ for 24 hours at $37^{\circ} \mathrm{C}$. After that, MTT ( $20 \mu \mathrm{M}$, Sigma) was added to each well and plates were incubated for $4 \mathrm{~h}$ at $37^{\circ} \mathrm{C}$. Dimethyl sulfoxide (150 $\mu$ l, Sigma) was then added to each well and the absorbance at $490 \mathrm{~nm}$ was recorded.

\section{Quantitative polymerase chain reaction array (qPCR)}

According to the manufacture's protocol, total RNA was extracted from cultured cardiomyocytes by RNeasy RNA extraction kit (Qiagen, Germany). Total $1 \mu \mathrm{g}$ of RNA was reverse transcribed using the RevertAid First Strand cDNA Synthesis Kit (Thermo Fisher Scientific). The cDNA obtained was subjected to quantitative polymerase chain reactions (qPCR) with the SYBR Green reagent (Roche, Indianapolis, USA). And the 
mouse primers were shown below. BETA-ACTIN, forward5'CATTGCTGACAGGATGCAGAAGG-3' , backward5'-

TGCTGGAAGGTGGACAGTGAGG-3'; Casp9, Forward sequence 5'GCTGTGTCAAGTTTGCCTACCC-3', Reverse sequence 5'CCAGAATGCCATCCAAGGTCTC-3' Casp3, Forward sequence 5'GGAGTCTGACTGGAAAGCCGAA-3', Reverse sequence 5'CTTCTGGCAAGCCATCTCCTCA-3'; Bax, Forward sequence 5'AGGATGCGTCCACCAAGAAGCT-3', Reverse sequence 5'TCCGTGTCCACGTCAGCAATCA-3'. Bcl-2, Forward sequence 5'AGCATGCGACCTCTGTTTGA-3', Reverse sequence 5'GCCACACGTTTCTTGGCAAT-3'. The relative mRNA expression levels were normalized to BETA-ACTIN.

\section{Statistical Analysis}

All results were performed at least three independent experiments and expressed as means \pm SEM. All of the statistical comparisons were made using a One-way or Twoway ANOVA post hoc test with Bonferroni correction to compare the groups. $\mathrm{P}<0.05$ was considered to be statistically significant. Analysis and graphs were performed using GraphPad Prism software 6.01 (San Diego, CA).

\section{Results}




\section{Isolation of exosome from CD90 positive cardiac fibroblasts}

The monolayers of primary neonatal cardiac fibroblasts adherent on the plates and showed spindle form. Almost all cardiac fibroblasts were positive expressed membrane receptor marker named fibroblast specific protein (FSP1, Fig 1A). Confocal imaging showed a moderate proportion of cardiac fibroblasts expressed CD90 (Fig 1B). The percentage of CD90 positive cardiac fibroblasts was about $35 \%$ when sorted by FACS(Fig 1C). CD90 positive cells gradually adherent to the dish within 24 hours and quickly amplified to a confluent monolayer within 2 days.

Next, we isolated exosomes from CD90 positive cells at passage 2 using standard differential and ultracentrifugation protocols. The exosome we isolated expressed the markers of high levels of CD63, CD81, TSG101, and showed low GRP94 expression (Fig 2A). Moreover, we found that the size of most exosome was between $100 \mathrm{~nm}$ and $150 \mathrm{~nm}$ (Fig 2B). Finally, TEM showed the typical electron micrographs of exosome (Fig 2C). Thus, the exosome from CD 90 positive cardiac fibroblasts was of highquality.

\section{Effects of exosome delivery on heart function of mice with myocardial infarction}

After ligation of the left ascending coronary artery, the electrography image showed myocardial ischemic morphology, which confirmed the success of the AMI model (Fig 3A). There was no death in the sham-operated groups, whereas 5 and 2 mice were dead 
in injury groups and exosome treated groups, respectively. The mortality rate of the injury group is about $50 \%$ at $30 \mathrm{~d}$ after MI, and the mortality of EXO group is about $20 \%$ at 30d after MI (Fig 3B). At 4 weeks after injury, the weight of the mice body and heart were measured (Fig 3C). The heart weight of exosome treated mice were significantly increased when compared with injury groups $(124.8 \mathrm{mg} \pm 1.4 \mathrm{mg} v s 114.5 \mathrm{mg} \pm 2.5 \mathrm{mg}$, $\mathrm{P}<0.05$ ). Similarly, the body weight of exosome treated mice were notably higher than that of injury mice $(32.0 \mathrm{~g} \pm 0.9 \mathrm{~g}$ vs $35.75 \mathrm{~g} \pm 0.8 \mathrm{~g}, \mathrm{P}<0.05)$. However, there was no prominent difference between them when compared the ratio of heart/body weight $(3.58 \pm 0.14$ vs $3.49 \pm 0.11, \mathrm{P}>0.05)$.

Heart functions were analyzed by echocardiography before sacrificed (Fig 4A), and parameters such as LVEF, LVFS, LVESD, LVEDD were analyzed (Fig 4B). Compared with injury groups, LVEF was significantly elevated in exosome delivery groups $(11.81 \%$ $\pm 0.95 \%$ vs $22.97 \% \pm 1.50 \%, \mathrm{P}<0.01)$. Also, there was a statistical difference in LVFS between the injury groups and exosome therapy groups $(5.829 \% \pm 0.71 \%$ vs $10.03 \% \pm$ $1.21 \%, \mathrm{P}<0.05)$. As for LVESD and LVEDD, the exosome treated groups had a significant restoration when compared to injury groups (LVESD: $4.45 \mathrm{~mm} \pm 0.07 \mathrm{~mm}$ vs $5.88 \mathrm{~mm} \pm 0.31 \mathrm{~mm}, \mathrm{P}<0.05$; LVEDD: $4.89 \mathrm{~mm} \pm 0.08 \mathrm{~mm}$ vs $5.85 \mathrm{~mm} \pm 0.15 \mathrm{~mm}$, $\mathrm{P}<0.01$ ). In summary, exosome delivery could improve the survival rate and heart function in mice with myocardial infarction injury. 


\section{Effects of exosome delivery on pathological changes of mice with myocardial infarction}

Hearts were collected at 4 weeks after surgery for pathological analysis using Immunohistochemically staining. As shown in figure 5A, all the apex and anterior left ventricular were pale stained, and the percentage of infarcted area in injury groups were more significant than that of exosome treated groups $(73.33 \% \pm 6.0 \%$ vs $23.33 \% \pm$ $4.4 \%, \mathrm{P}<0.01$ ). The myocardial fibers were ruptured and lost in the infarction zone (Fig 5B). Compared with exosome treated mice, the deposit of funicular collagen fibers in the infarction scar zone was more severe in the injured groups $(56.0 \% \pm 3.05 \%$ vs $82.33 \%$ $\pm 5.04 \%, \mathrm{P}<0.05)($ Fig $5 \mathrm{C})$. In short, exosome delivery to myocardial infarction mice was able to reduce remodeling of the ventricle.

\section{Effects of exosome delivery on apoptosis changes of mice with myocardial infarction}

To measure the anti-apoptosis effect of exosome in vivo, the proteins associated with apoptosis, such as cleaved caspase 3, cleaved caspase 9, and Bax, were highly expressed in exosome treated groups (Fig 6A). Meanwhile, the anti-apoptosis related protein $(\mathrm{Bcl}-2)$ were low expressed in the exosome therapy groups. Therefore, this data indicated that the apoptosis of heart tissues was attenuated by exosome therapy after myocardial infarction. 


\section{Exosome incubation attenuated apoptosis of cardiomyocytes in vitro}

To assess the effects of exosomes in vitro, cardiomyocytes were incubated with exosome containing medium under hypoxia condition. Firstly, we used MTT assay to measure the suitable concentration of exosome after injury in vitro, and the data showed that the viability of cardiomyocytes were significantly improved at the concentration of $5 \times 10^{3} / \mathrm{ML}$, and this benefit was not remarkably increased when the concentration were elevated. When exosome was introduced during hypoxia, the percentage of apoptosis in cardiomyocytes were significantly decreased when compared to injury controls $(26.8 \%$ $\pm 2.9 \%$ vs $56.6 \% \pm 3.1 \%, \mathrm{P}<0.001)$.

Exosome inhibited apoptosis of the injured cardiomyocytes in vitro likely via PI3K/AKT signaling pathway

To measure the molecular mechanism of protective function from exosome, we investigated whether exosome from CD90 positive cardiomyocytes influenced PI3K/AKT signaling pathway, which played an important role in apoptosis of cells after injury. We found that the PI3k/AKT signaling pathway related protein p-PI3K and pAKT were significantly increased after exosome treatment in injury model (Fig 8A-C). However, this result only reflected the correlation between exosome and PI3K/AKT pathway. Thus, we used PI3K specific inhibitor (LY294002) to clarify the relationship between exosome and PI3K/AKT signaling pathway. The Q-PCR data showed that there was no statistical difference of apoptosis-related factors in the LY294002 
treatment group when compared with exosome group (Fig 8 D-F). In summary, exosome could protect cardiomyocytes under hypoxia conditions, likely via PI3K/AKT pathway.

\section{Discussion}

In this study, we investigated the pathological role of apoptosis after cardiomyocytes injury both in vivo and in vitro. We found the exosome from CD90 positive cardiac fibroblast enhanced the recovery of cardiac function by inhibiting apoptosis. Therefore, we investigated the mechanisms of the cardiac protective effects of exosome after hypoxic injury in vitro. We found that the exosome from CD90 positive cardiac fibroblasts induced recovery of cardiac function and reduced cardiac damage as well as fibrosis area in MI model via activation of the PI3K/AKT signaling pathway. We aimed to provide an evidence that the cardiac protective effects of exosome from CD90 positive cardiac fibroblast inhibited cardiac apoptosis after MI both in vivo and in vitro.

The heterogeneity was existed in cardiac fibroblasts, which may cause a diversity of therapeutic efficiency among the individuals(23, 24). CD90, a cell surface adhesion molecular, played a key role in the process of adhesion, proliferation, migration and apoptosis(25-27). Accordingly, increasing researches have demonstrated that the CD90 positive fibroblasts were important in the modulation of an inflammatory response, fibrosis during the process of tissue development or repair(28, 29). Moreover, CD90 positive fibroblasts, which represented the principal primary fibroblasts in myocardium 
exhibited promising repair potential after myocardial infarction by regulating the activity of cardiomyocytes(16). Similarly, our results confirmed the high ratio of CD90 positive cells among the primary cardiac fibroblasts.

The exosome is a kind of small vesicles and contained proteins and complex RNA, which formed by intracellular lysosomal microsome invagination from polyvesicles(30). During the pathological or physiological processes, exosome from different kinds of cells like stem cells, fibroblasts, fat cells or neurons can be released into the extracellular matrix and maintain microenvironment homeostasis(31, 32). Several studies have reported that the exosome from stem cells, fibroblasts, fat cells or macrophages can reduce myocardial injury and enhance cardiac function after MI in vivo and in vitro $(33,34)$. Similarly, our results showed that the exosome from CD90 positive fibroblasts could enhance cardiac function, decreased myocardial injury and fibrotic area after MI in vivo. Thus, these data demonstrated that exosomes were beneficial for the recovery of MI. As other studies reported that exosome promotes myocardial repair possibly related to inhibit myocardial apoptosis $(35,36)$. Therefore, we tested whether exosome derived from CD90 positive fibroblasts decreased the level of apoptosis in heart after MI. We found the apoptosis-related protein Cleaved Caspase 3, Cleaved Caspase 9, BAX was inhibited, and BCL-2 was induced after exosome treatment in MI model. These data showed CD90 positive fibroblasts exosome could inhibit apoptosis in cardiomyocytes after MI. As we know, the action of apoptotic inhibition effect mainly implemented through binding to target cell membrane proteins 
and sequentially activated signal pathways in target cells or exosome membrane proteins that could be cleaved by proteases. The cleaved fragments acted as ligands that binding to receptors on cell membranes, thus activated intracellular signaling pathways(37).

In this study, we have not studied how exosomes acted on receptors of myocardial membranes, and the work will be conducted in the future. Importantly, we focused on the signaling pathway modulation of cardiac myocytes induced by exosomes after MI. Therefore, we tested whether CD90 positive fibroblasts exosome can change apoptosisrelated signaling pathway in primary cardiomyocyte after injury in vitro. PI3K/AKT signaling pathway was a fundamental mechanism associated with apoptosis, cell death, autophagy(38-40). In brief, activation of PI3K/AKT signaling pathway could inhibit apoptosis of cardiomyocytes after injury(41). Here, we tested the effect of the exosome on PI3K/AKT pathway. Our data showed that the exosome from CD90 positive fibroblasts could induce PI3K/AKT pathway. Moreover, after blocked by PI3K specific inhibitors, the therapeutic effect of exosome on cardiomyocyte apoptosis significantly decreased. Their data indicated that exosome of CD90 positive fibroblasts could inhibit apoptosis of cardiomyocytes likely via PI3K/AKT signaling pathway after injury in vitro.

\section{Conclusion}


In summary, this study has shown that exosome from CD90 positive fibroblasts can improve cardiac function and attenuate fibrosis of mice with MI by decreasing apoptosis. Additionally, the exosome was able to increase the viability and reduce apoptosis in primary cardiomyocytes under hypoxia conditions probably via modulation of the PI3K/AKT signaling pathway. Therefore, exosome of CD90 positive fibroblasts is implicated as a potential therapeutic agent in MI.

\section{Acknowledgements}

This project was supported by the Research on science and technology of Liaoning Provincial Department of Education.

\section{Declarations}

All authors declare no financial conflict of interest.

\section{Ethics approval and consent to participate}

All surgical procedures and animal care protocols in the present study were approved by the Laboratory Animal Research Committee of Jinzhou Medical University.

\section{Consent for publication}

Not applicable. 


\section{Availability of data and material}

All the data generated during this study are included in this published article.

\section{Funding}

This study was supported by the China Postdoctoral Science Foundation (Project Numbers: 2020M683126).

\section{Authors' contributions}

XDM and LYL conceived and designed the study, revised the manuscript, and wrote

the manuscript. LQ performed most of the cellular experiments. SXL helped to perform the flow cytometry experiments and collected the animal samples. WS analyzed the data and made the figures. All authors read and approved the final manuscript.

\section{Abbreviations}

MI: Myocardial infarction; PI3K :phosphatidylinositol 3-kinase; AKT: protein kinase B ; EXO: exosome; TEM: transmission electron microscope; LVEF: Left ventricular ejection fraction; LVFS: Left ventricular fractional shortening; LV: Left ventricle; LVESD: Left ventricular end-systolic diameter; LVEDD: Left ventricular end-diastolic diameter; TTC: Triphenylterazolium chloride; MTT: 3-(4,5-Dimethylthiazol-2-yl)-2,5- 
diphenyltetrazolium bromide; qPCR: quantitative polymerase chain reactions; FSP1: fibroblast specific protein;

\section{Reference}

1. Levine GN, Bates ER, Bittl JA, et al. 2016 ACC/AHA Guideline Focused Update on Duration of Dual Antiplatelet Therapy in Patients With Coronary Artery Disease: A Report of the American College of Cardiology/American Heart Association Task Force on Clinical Practice Guidelines: An Update of the 2011 ACCF/AHA/SCAI Guideline for Percutaneous Coronary Intervention, 2011 ACCF/AHA Guideline for Coronary Artery Bypass Graft Surgery, 2012 ACC/AHA/ACP/AATS/PCNA/SCAI/STS Guideline for the Diagnosis and Management of Patients With Stable Ischemic Heart Disease, 2013 ACCF/AHA Guideline for the Management of ST-Elevation Myocardial Infarction, 2014 AHA/ACC Guideline for the Management of Patients With Non-STElevation Acute Coronary Syndromes, and 2014 ACC/AHA Guideline on Perioperative Cardiovascular Evaluation and Management of Patients Undergoing Noncardiac Surgery. Circulation 134: e123-155, 2016.

2. El Salcry SA, Fayez D, Morad CS, et al.: Ischemic heart disease and rheumatoid arthritis: Do inflammatory cytokines have a role? Cytokine 96: 228-233, 2017.

3. Giuseppe C, Paul J and Hans-Ulrich I: Use of nitrates in ischemic heart disease. Expert Opin Pharmaco 16: 1567-1572, 2015.

4. Shiba Y, Gomibuchi T, Seto T, et al.: Allogeneic transplantation of iPS cell-derived cardiomyocytes regenerates primate hearts. Nature 538: 388-+, 2016.

5. Yoshida Y and Yamanaka S: Induced Pluripotent Stem Cells 10 Years Later For Cardiac Applications. Circ Res 120: 1958-1968, 2017.

6. Xie D, Liao Y, Wu B, et al.: Cardiac Nestin(+) Cells Derived from Early Stage of Dilated Cardiomyopathy Enhanced the Survival of the Doxorubicin-Injured Cardiac Muscle HL-1 Cells. Int Heart J 59: 180-189, 2018. 
7. Li SH, Sun L, Yang L, et al.: Young Bone-Marrow Sca-1(+) Stem Cells Rejuvenate the Aged Heart and Improve Function after Injury through PDGFRbeta-Akt pathway. Sci Rep 7: 41756, 2017.

8. Alvarez R, Lee HL, Hong C and Wang CY: Single CD271 marker isolates mesenchymal stem cells from human dental pulp. Int J Oral Sci 7: 205-212, 2015.

9. Pinho S, Lacombe J, Hanoun M, et al.: PDGFRalpha and CD51 mark human nestin+ sphere-forming mesenchymal stem cells capable of hematopoietic progenitor cell expansion. J Exp Med 210: 1351-1367, 2013.

10. Gourdie RG, Dimmeler S and Kohl P: Novel therapeutic strategies targeting fibroblasts and fibrosis in heart disease. Nat Rev Drug Discov 15: 620-638, 2016.

11. Tallquist MD and Molkentin JD: Redefining the identity of cardiac fibroblasts. Nat Rev Cardiol 14: 484-491, 2017.

12. Porter KE and Turner NA: Cardiac fibroblasts: At the heart of myocardial remodeling. Pharmacol Therapeut 123: 255-278, 2009.

13. Swonger JM, Liu JS, Ivey MJ and Tallquist MD: Genetic tools for identifying and manipulating fibroblasts in the mouse. Differentiation 92: 66-83, 2016.

14. Felley-Bosco E and Leyton L: Editorial: Thy1/CD90 Surface Glycoprotein: Sensor of Microenvironment? Front Cell Dev Biol 7: 162, 2019.

15. Martini H, Iacovoni JS, Maggiorani D, et al.: Aging induces cardiac mesenchymal stromal cell senescence and promotes endothelial cell fate of the CD90 + subset. Aging Cell 18: e13015, 2019.

16. Chang Y, Li C, Jia Y, et al.: CD90(+) cardiac fibroblasts reduce fibrosis of acute myocardial injury in rats. Int J Biochem Cell Biol 96: 20-28, 2018.

17. Ludwig N, Whiteside TL and Reichert TE: Challenges in Exosome Isolation and Analysis in Health and Disease. Int J Mol Sci 202019.

18. Rezaie J, Rahbarghazi R, Pezeshki M, et al.: Cardioprotective role of extracellular 
vesicles: A highlight on exosome beneficial effects in cardiovascular diseases. J Cell Physiol 234: 21732-21745, 2019.

19. Suzuki E, Fujita D, Takahashi M, Oba S and Nishimatsu H: Therapeutic Effects of Mesenchymal Stem Cell-Derived Exosomes in Cardiovascular Disease. Adv Exp Med Biol 998: 179-185, 2017.

20. Abrial M, Da Silva CC, Pillot B, et al.: Cardiac fibroblasts protect cardiomyocytes against lethal ischemia-reperfusion injury. J Mol Cell Cardiol 68: 56-65, 2014.

21. Frangogiannis NG: Cardiac fibrosis: Cell biological mechanisms, molecular pathways and therapeutic opportunities. Mol Aspects Med 65: 70-99, 2019.

22. Cosme J, Guo H, Hadipour-Lakmehsari S, Emili A and Gramolini AO: HypoxiaInduced Changes in the Fibroblast Secretome, Exosome, and Whole-Cell Proteome Using Cultured, Cardiac-Derived Cells Isolated from Neonatal Mice. J Proteome Res 16: 2836-2847, 2017.

23. Doppler SA, Carvalho C, Lahm H, et al.: Cardiac fibroblasts: more than mechanical support. J Thorac Dis 9: S36-S51, 2017.

24. Roche PL, Filomeno KL, Bagchi RA and Czubryt MP: Intracellular Signaling of Cardiac Fibroblasts. Compr Physiol 5: 721-760, 2015.

25. Zhu GC, Gao L, He JY, et al.: CD90 is upregulated in gastric cancer tissues and inhibits gastric cancer cell apoptosis by modulating the expression level of SPARC protein. Oncology Reports 34: 2497-2506, 2015.

26. Avril T, Etcheverry A, Pineau R, et al.: CD90 Expression Controls Migration and Predicts Dasatinib Response in Glioblastoma. Clin Cancer Res 23: 7360-7374, 2017.

27. Song XH, Hong CY, Zheng QQ, et al.: Differentiation potential of rabbit CD90positive cells sorted from adipose-derived stem cells in vitro. In Vitro Cell Dev-An 53: 77-82, 2017.

28. Cohen PY, Breuer R, Zisman P and Wallach-Dayan SB: Bleomycin-Treated Chimeric Thy1-Deficient Mice with Thy1-Deficient Myofibroblasts and Thy-Positive 
Lymphocytes Resolve Inflammation without Affecting the Fibrotic Response. Mediat Inflamm2015.

29. Chen J, Li HY, Wang D and Guo XZ: Delphinidin protects beta2m-/Thy1+ bone marrow-derived hepatocyte stem cells against TGF-beta1-induced oxidative stress and apoptosis through the PI3K/Akt pathway in vitro. Chem Biol Interact 297: 109-118, 2019.

30. Yang F, Liao XZ, Tian Y and Li GY: Exosome separation using microfluidic systems: size-based, immunoaffinity-based and dynamic methodologies. Biotechnol J 122017.

31. Milane L, Singh A, Mattheolabakis G, Suresh M and Amiji MM: Exosome mediated communication within the tumor microenvironment. J Control Release 219: 278-294, 2015.

32. Teng XM, Chen L, Chen WQ, Yang JJ, Yang ZY and Shen ZY: Mesenchymal Stem Cell-Derived Exosomes Improve the Microenvironment of Infarcted Myocardium Contributing to Angiogenesis and Anti-Inflammation. Cell Physiol Biochem 37: 2415$2424,2015$.

33. Zhang Y, Hu YW, Zheng L and Wang Q: Characteristics and Roles of Exosomes in Cardiovascular Disease. DNA Cell Biol 36: 202-211, 2017.

34. Adamiak $M$ and Sahoo S: Exosomes in Myocardial Repair: Advances and Challenges in the Development of Next-Generation Therapeutics. Mol Ther 26: 1635$1643,2018$.

35. Xiao J, Pan Y, Li XH, et al.: Cardiac progenitor cell-derived exosomes prevent cardiomyocytes apoptosis through exosomal miR-21 by targeting PDCD4. Cell Death \& Disease 72016.

36. Jung JH, Fu XB and Yang PC: Exosomes Generated From iPSC-Derivatives New Direction for Stem Cell Therapy in Human Heart Diseases. Circ Res 120: 407-417, 2017. 
37. Zhang SP, Chuah SJ, Lai RC, Hui JHP, Lim SK and Toh WS: MSC exosomes mediate cartilage repair by enhancing proliferation, attenuating apoptosis and modulating immune reactivity. Biomaterials 156: 16-27, 2018.

38. Khan MA, Jain VK, Rizwanullah M, Ahmad J and Jain K: PI3K/AKT/mTOR pathway inhibitors in triple-negative breast cancer: a review on drug discovery and future challenges. Drug Discov Today2019.

39. Li YL, Lin S, Xu C, Zhang P and Mei XF: Triggering of Autophagy by Baicalein in Response to Apoptosis after Spinal Cord Injury: Possible Involvement of the PI3K Activation. Biological \& Pharmaceutical Bulletin 41: 478-486, 2018.

40. Wei X, Luo L and Chen J: Roles of mTOR Signaling in Tissue Regeneration. Cells 82019.

41. Li Y, Guo Y, Fan Y, Tian H, Li K and Mei X: Melatonin Enhances Autophagy and Reduces Apoptosis to Promote Locomotor Recovery in Spinal Cord Injury via the PI3K/AKT/mTOR Signaling Pathway. Neurochem Res 44: 2007-2019, 2019. 


\section{Figure legends}

Figure 1. Isolation of CD90 positive cardiac fibroblast. A. FSP1 (red) immunofluorescent staining of cardiac fibroblasts, nucleus was stained by DAPI (blue), Scale bar, $200 \mu \mathrm{m}$. B. CD90 (green) immunofluorescent staining of cardiac fibroblasts, nucleus was stained by DAPI (blue), Scale bar, $100 \mu \mathrm{m}$. C. CD90 positive cardiac fibroblasts were isolated by flow cytometry. Images of cultured CD90 positive cells were presented by light microscope, scale bar, $200 \mu \mathrm{m}$. FSP1, fibroblasts specific protein 1.

Figure 2. Characterization of exosome derived from CD90 positive cardiac fibroblasts exosome. A. Assessment of CD63, CD81, TSG101 and GRP94 expression in exosome by western blot. B. Size distribution of particles among exosomes using NanoSight imaging. C. Electron microscopy images of exosome. Scale bar, 100nm.

Figure 3. Comparisons of survival, heart weight and body weight. A. Electrography image of heart before myocardial infarction and after myocardial infarction. B. Survival curves of mice after injury. C. Analysis of heart weight, body weight and Heart/body weight ratio. Data are shown as mean $\pm \mathrm{SEM}, *<0.05, \mathrm{~ns}>0.05$ compared with injury groups.

Figure 4. The effects of exosome on heart function after injury. Heart functions were analyzed at 4 weeks after injury. A. ultrasonic echocardiography images of hearts, 
yellow line, end of diastole, red line, end of systole. B. Comparison of LVEF, LVFS, LVESD, LVEDD in each group by echocardiography. Data are shown as mean $\pm \mathrm{SEM}$, $* \mathrm{p}<0.05, * * \mathrm{p}<0.01$ compared to injury groups.

Figure 5. The pathological regulatory effects of exosome on heart after myocardial infarction. Hearts were collected at 4 weeks after surgery. A. The presentative images of TTC stain in hearts, viable heart muscles were red-stained, infarction tissues were pale, ischemic ratio was presented as a percentage of total area of left ventricular using the middle heart section. B. Images of hearts by HE stains, scale bar, $40 \mu \mathrm{m}$. C. cardiac fibrosis was represented by Masson stain, the fibrosis area ratio was determined by a percentage of area per the high-power field, scale bar, $40 \mu \mathrm{m}$. Data are shown as mean $\pm \mathrm{SEM}, * \mathrm{p}<0.05, * * \mathrm{p}<0.01$ compared to injury groups.

Figure 6. The apoptotic effects of exosome on heart after myocardial infarction. Heart tissues were collected at day 3 post injury. A. Representative western blot images of cleaved caspase 3, cleaved caspase 9, BAX BCL-2 and BETA-ACTIN from heart tissues. B-D. Comparison of cleaved caspase 3, cleaved caspase 9 and BAX/BCL-2, protein expression was normalized to BETA-ACTIN. Data are shown as mean $\pm \mathrm{SEM}$, $* \mathrm{p}<0.05, * * \mathrm{p}<0.01$ compared to injury groups.

Figure 7. The effects of exosome on cardiomyocytes after hypoxia in vitro. A. MTT viability data of cardiomyocytes under hypoxia condition with or without exosome coincubation, Absorbance data were analyzed via one-way ANOVA and posthoc Tukey's 
test. Data are shown as mean $\pm \operatorname{SEM}$ (ns $\mathrm{p}>0.05, * \mathrm{p}<0.05, * * \mathrm{p}<0.01$ compared to exosome-free co-incubation). B. Apoptosis detection in cardiomyocytes under hypoxia condition by immunofluorescence. red, Tunel; green $\alpha$-actinin, blue, DAPI. C. Comparison of a percentage of tunel positive cells. Data are shown as mean $\pm \mathrm{SEM}, * * * \mathrm{p}<$ 0.001 compared to injury groups.

Figure 8. Exosome co-incubation decreased apoptosis of myocardiocytes induced by hypoxia via PI3K/AKT signaling pathway. A. Assessment of $\mathrm{p}-\mathrm{PI} 3 \mathrm{~K}, \mathrm{p}-\mathrm{AKT}$ and BETA-ACTIN expression in cardiomyocytes in vitro by western blot. B-C. Analyze of p-PI3K and p-AKT expression, and protein expression was normalized to BETAACTIN, respectively. Data are shown as mean \pm SEM, $* \mathrm{p}<0.05, * * \mathrm{p}<0.01$ compared to injury groups. D-F. Relative mRNA expression of cleaved caspase 3, cleaved caspase 9 and BAX/BCL-2 in cardiomyocytes under hypoxia condition with or without supplement of LY294002, gene expression was normalized to BETA-ACTIN. Data are shown as mean \pm SEM, ns $\mathrm{p}>0.05, * \mathrm{p}<0.05, * * \mathrm{p}<0.01$. 
Figures:

Figure 1
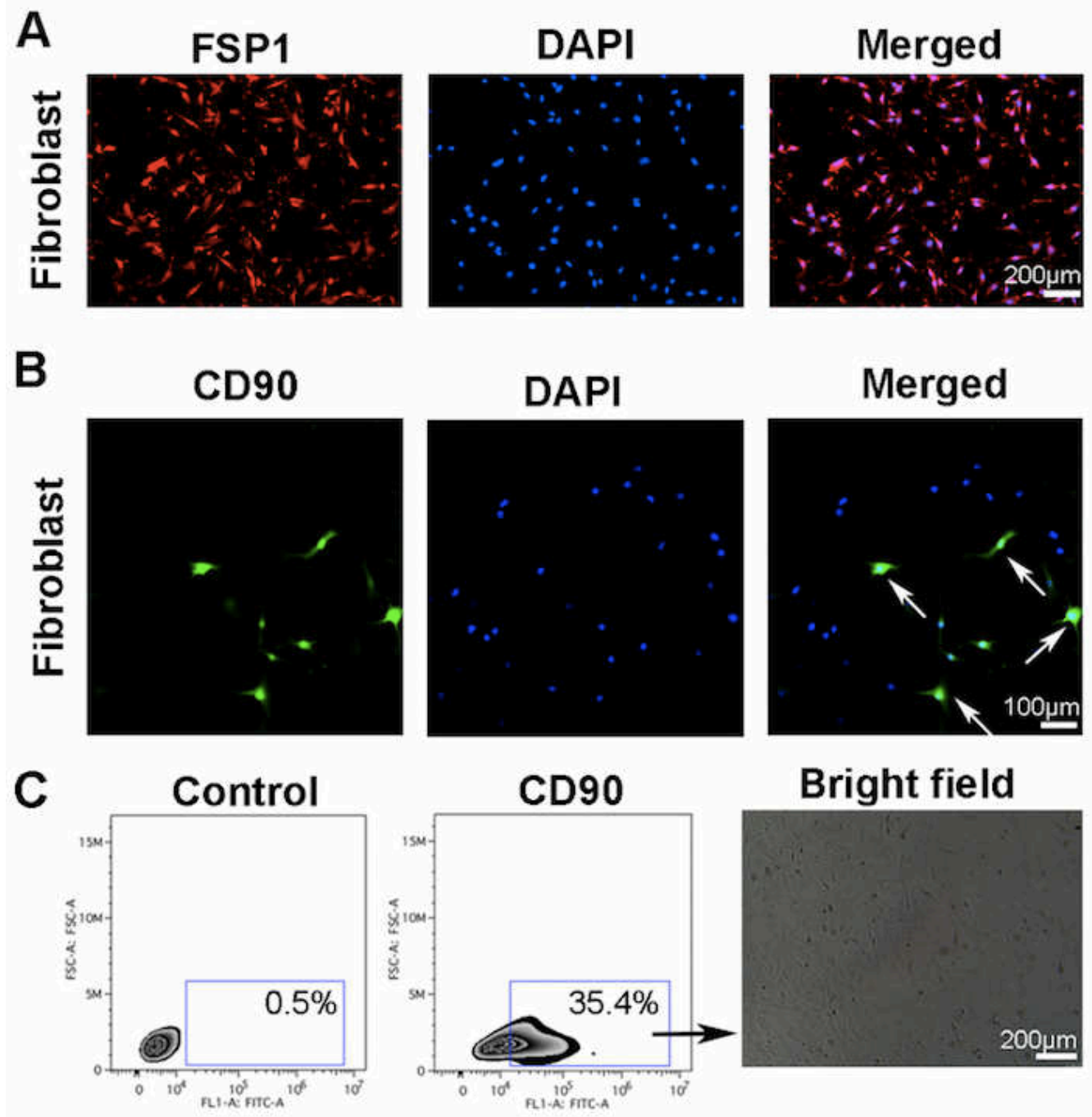

Bright field

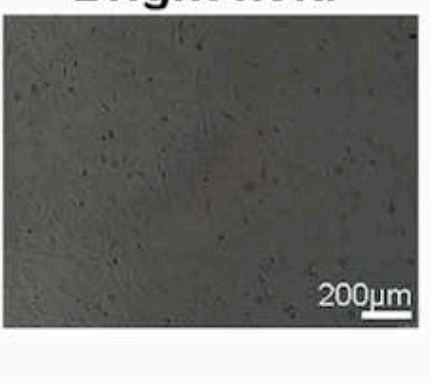

Figure 1. Isolation of CD90 positive cardiac fibroblast. 
Figure 2
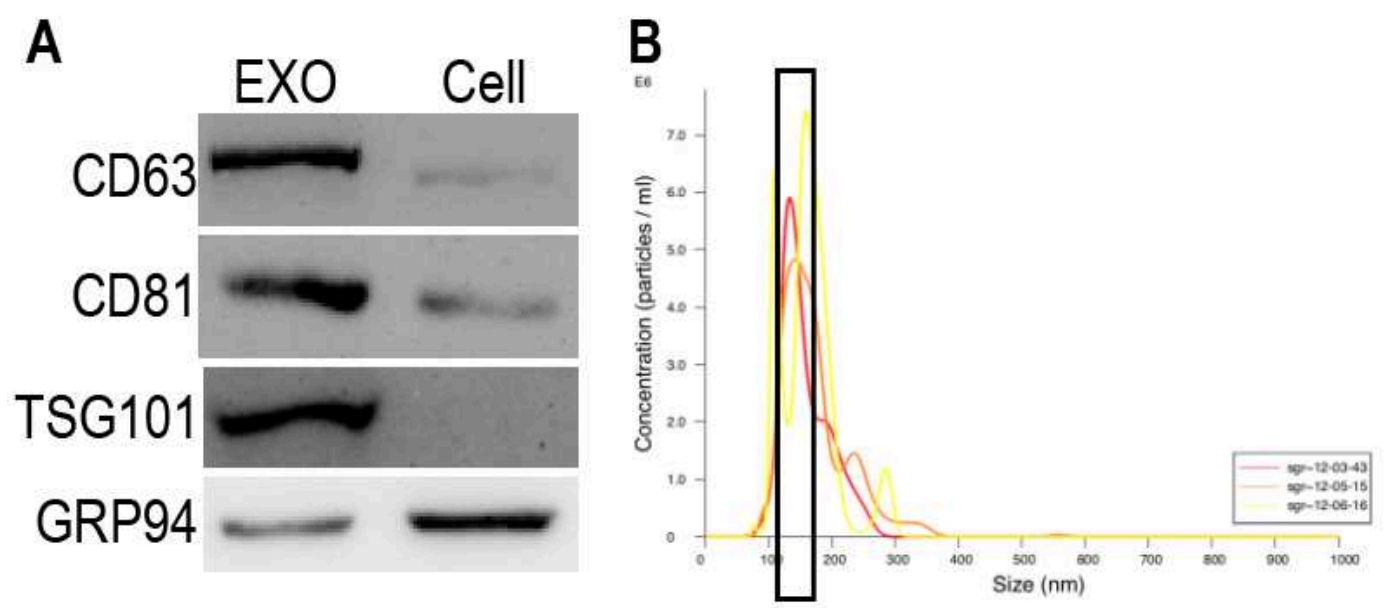

C

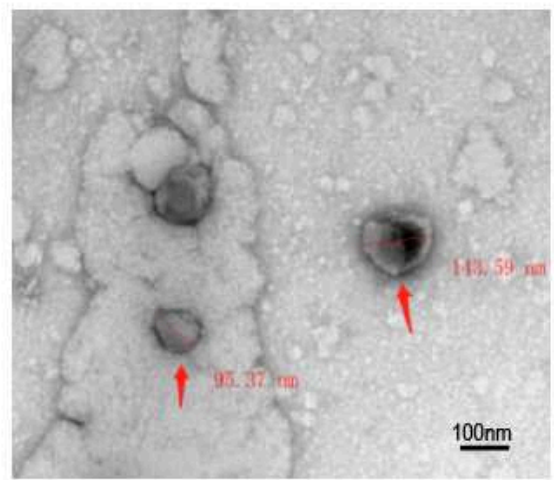

Figure 2. Characterization of exosome derived from CD90 positive cardiac fibroblasts exosome. 
Figure 3

A

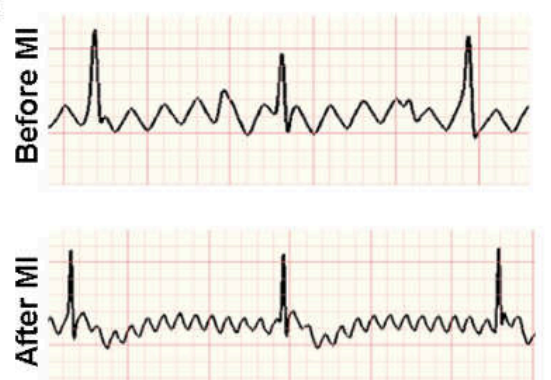

B

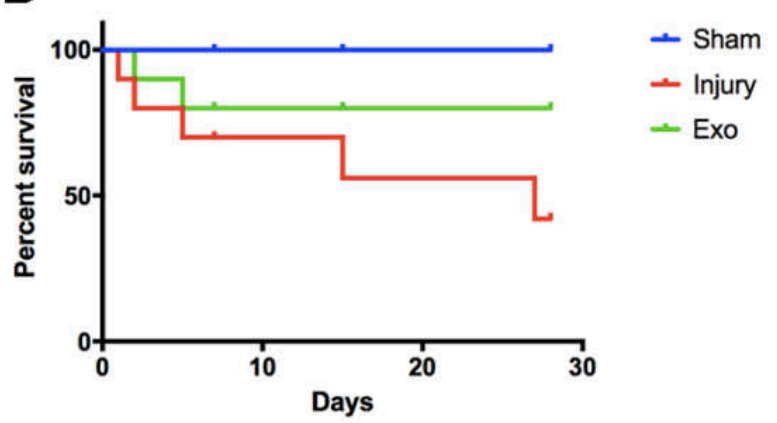

C

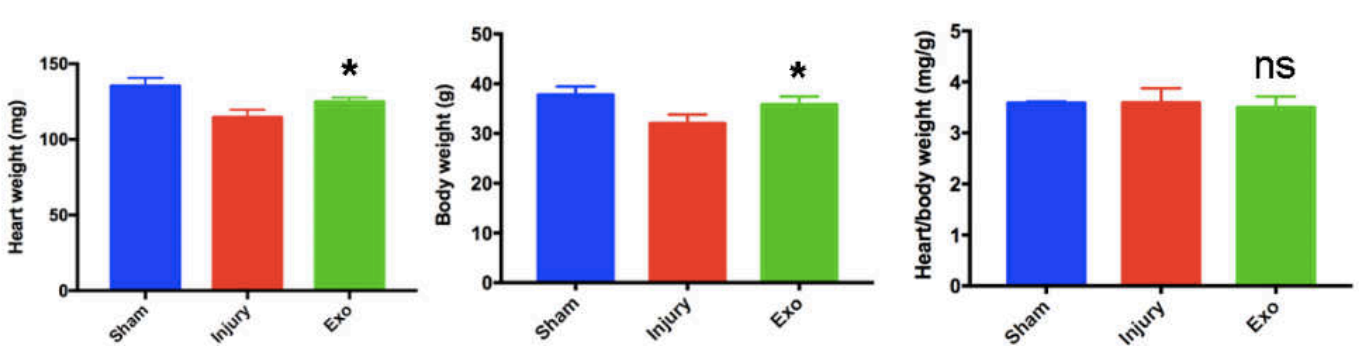

Figure 3. Comparisons of survival, heart weight and body weight. 
Figure 4
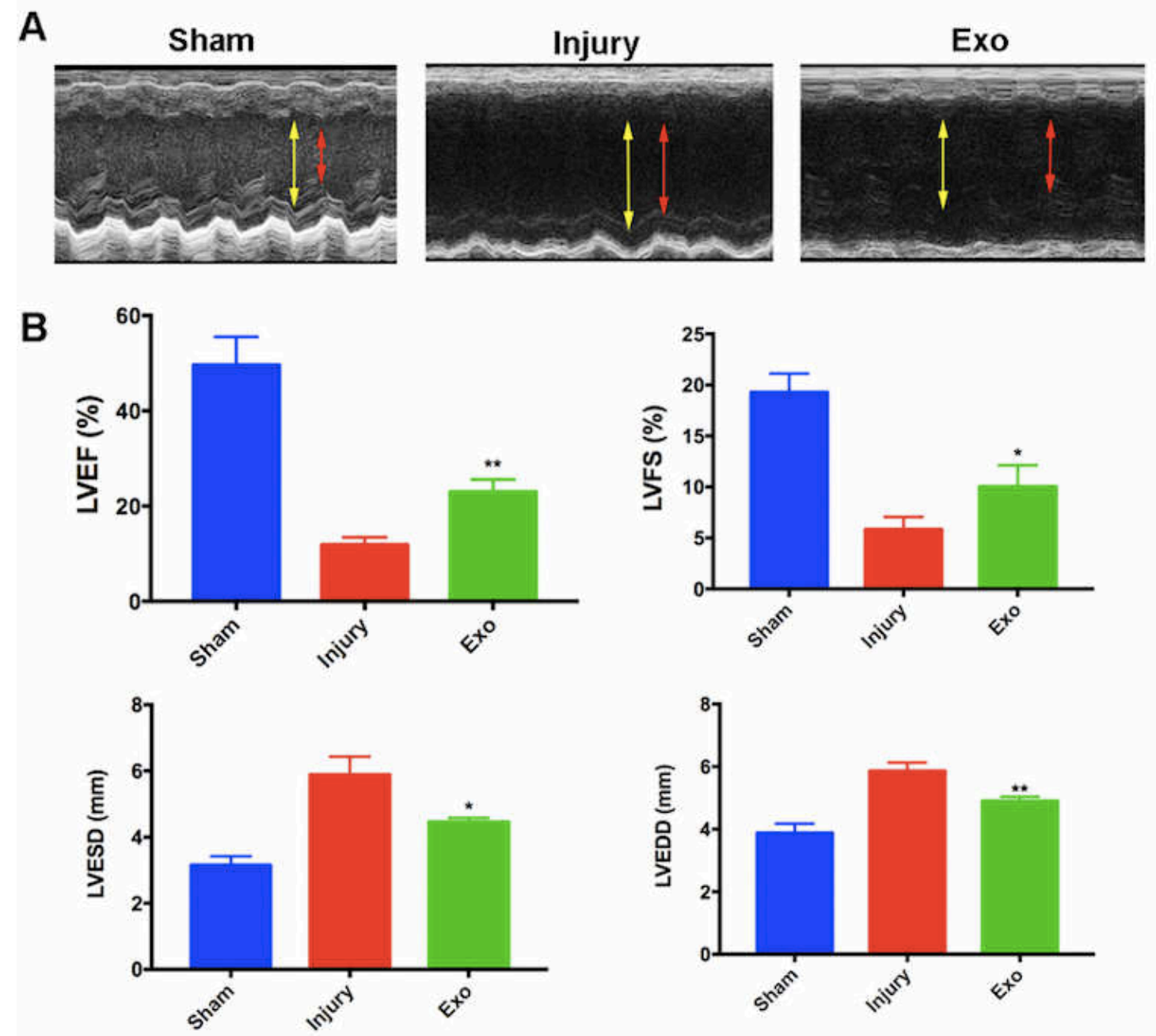

Figure 4. The effects on exosome on heart function after injury. 
Figure 5
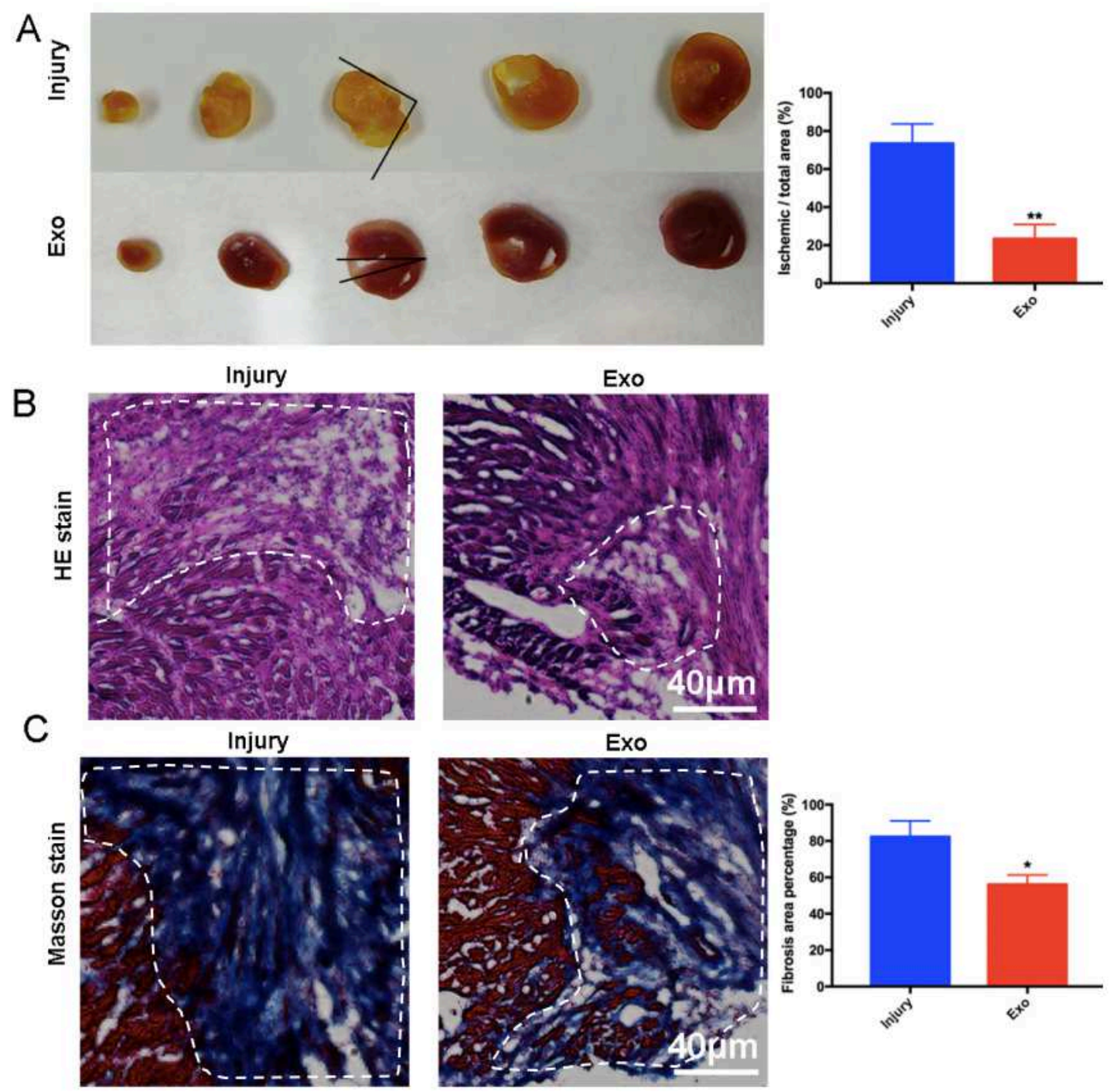

Figure 5. The pathological regulatory effects of exosome on heart after myocardial infarction. 
Figure 6

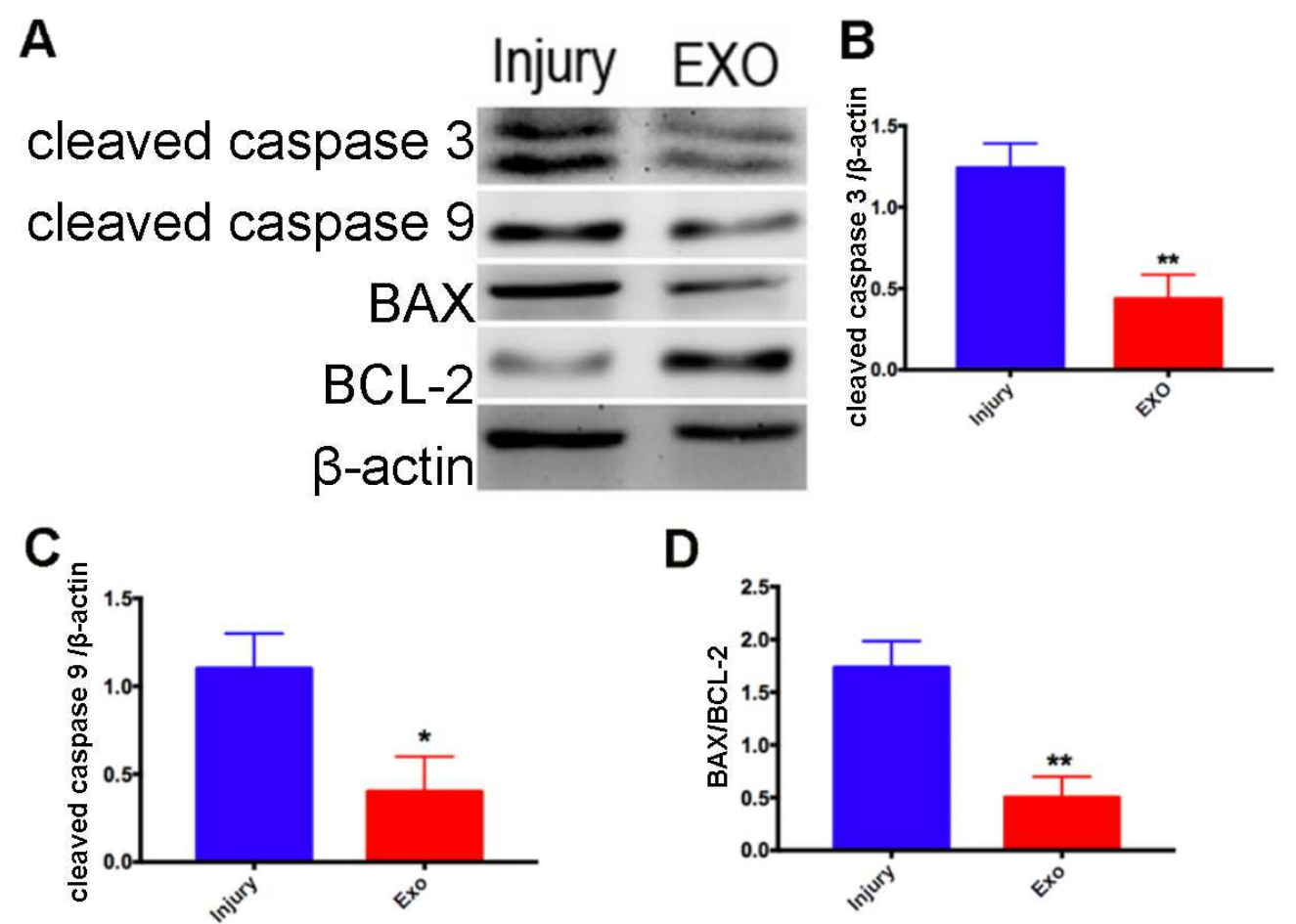

Figure 6. The apoptotic effects of exosome on heart after myocardial infarction. 
Figure 7

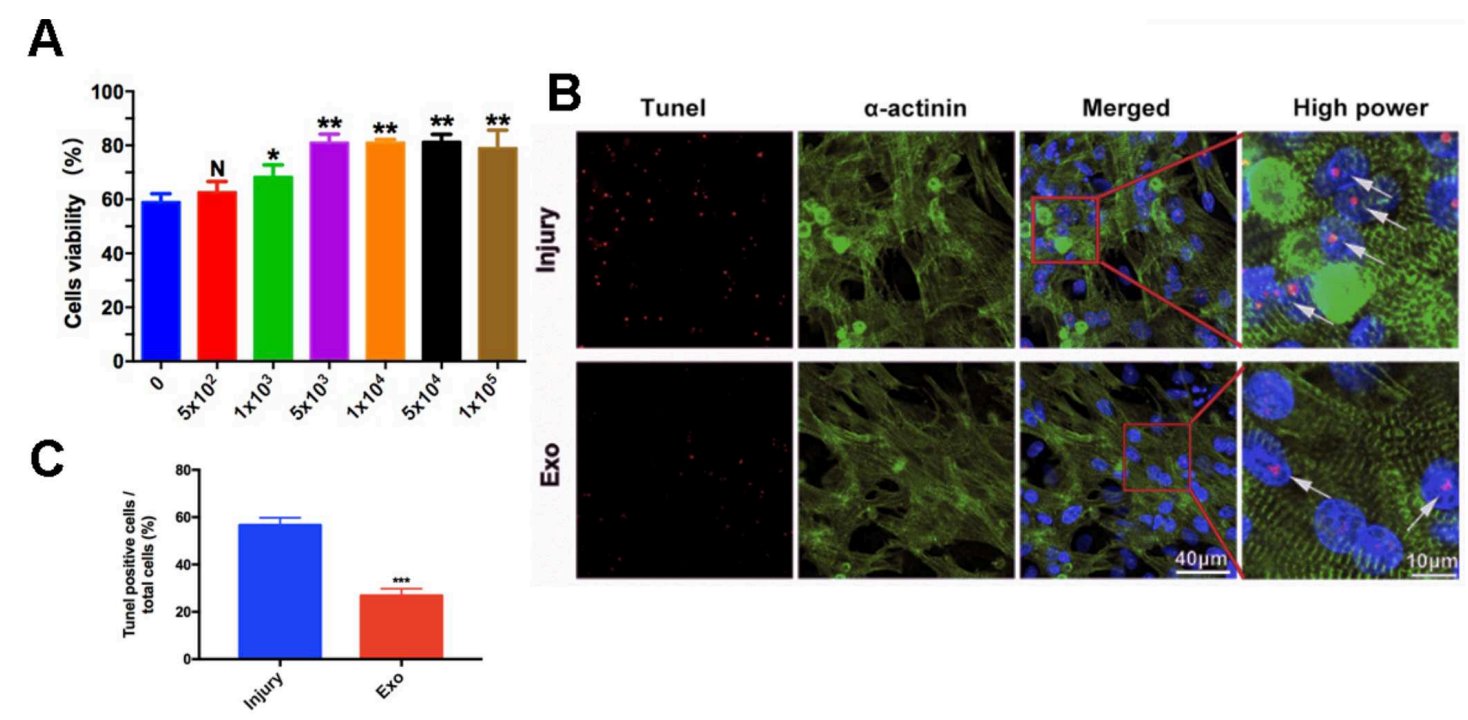

Figure 7. The effects of exosome on cardiomyocytes after hypoxia in vitro. 
Figure 8

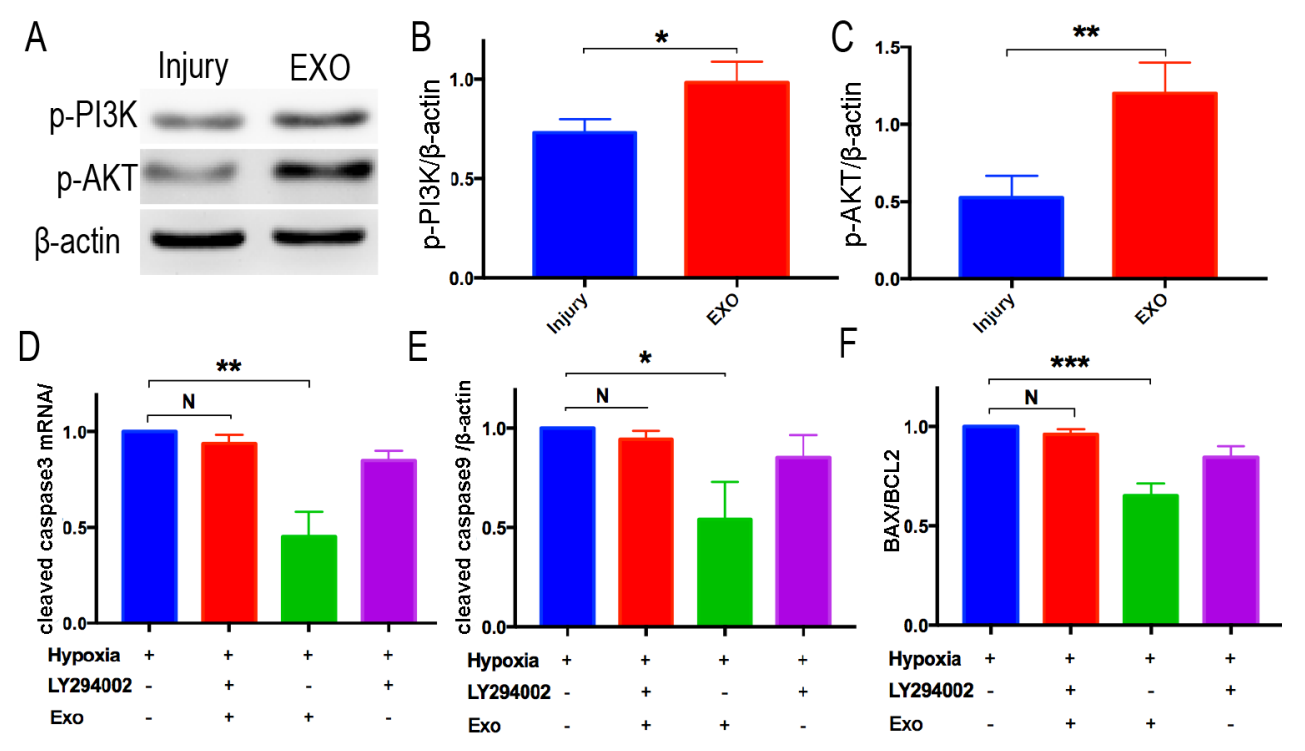

Figure 8. Exosome co-incubation decreased apoptosis of cardiomyocytes induced by hypoxia via PI3K/AKT signaling pathway. 


\section{Figures}
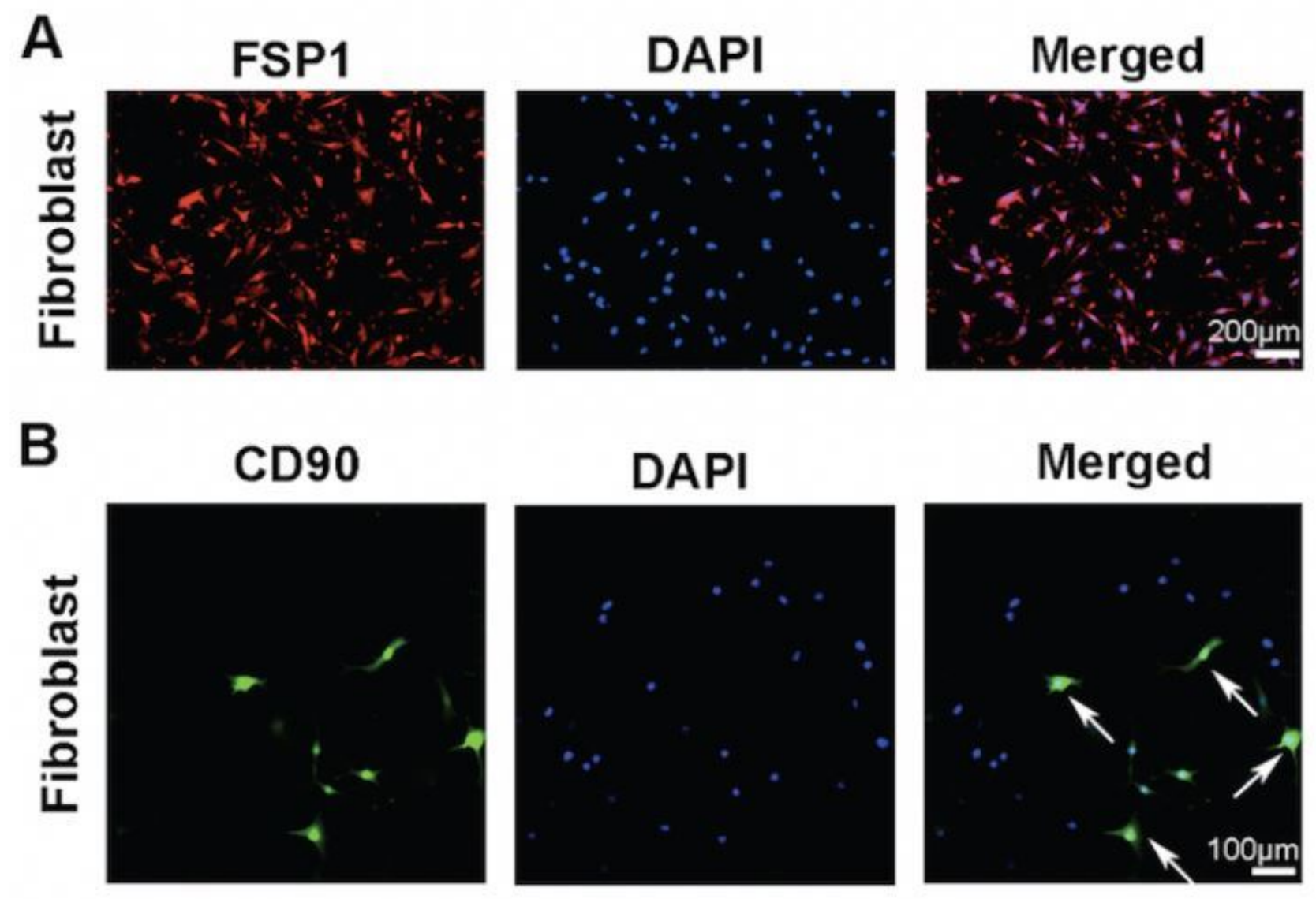

\section{Merged}
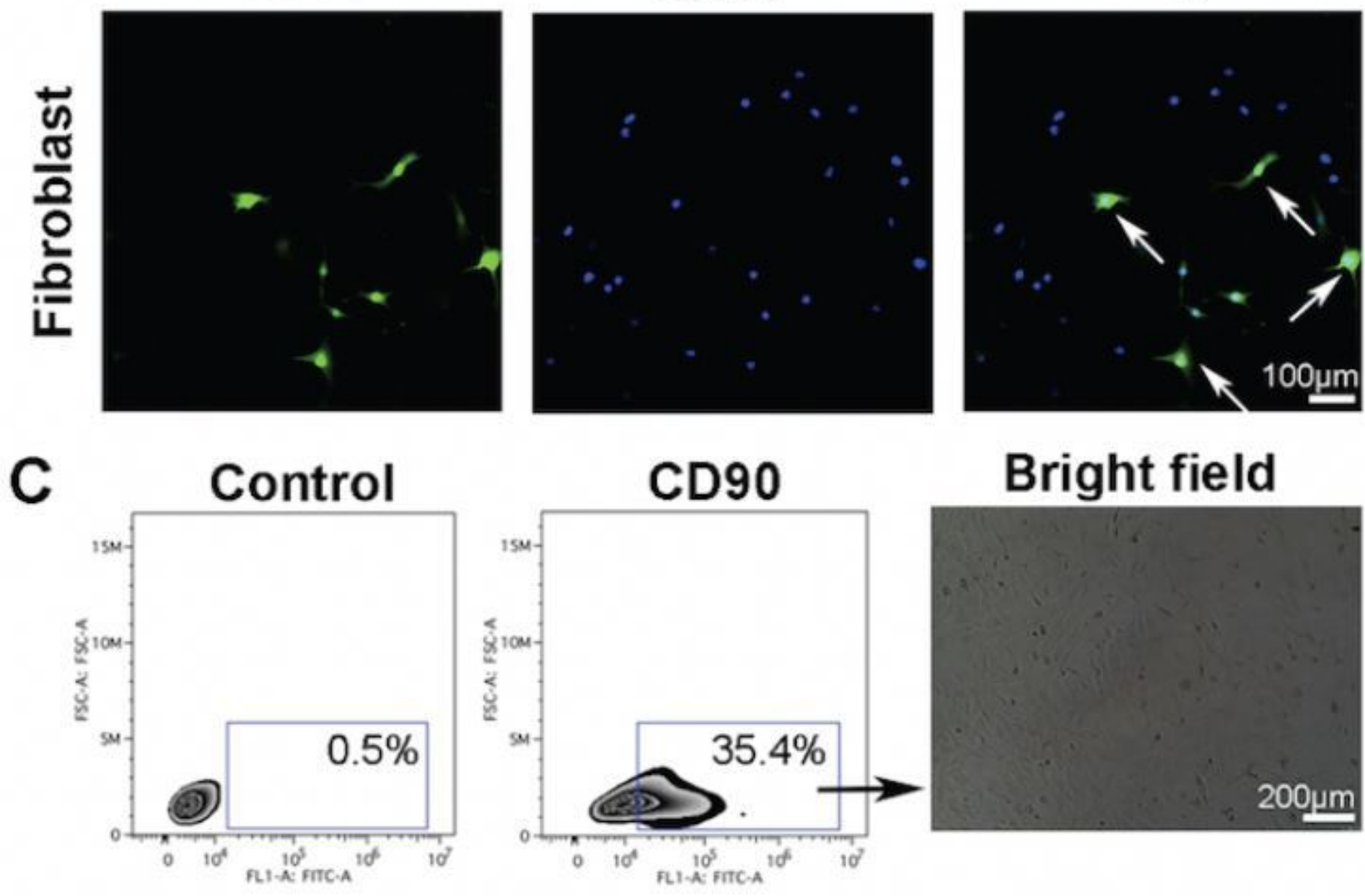

Bright field

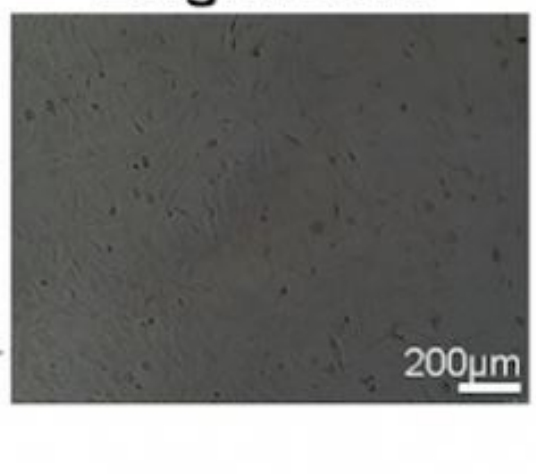

Figure 1

Isolation of CD90 positive cardiac fibroblast. A. FSP1 (red) immunofluorescent staining of cardiac fibroblasts, nucleus was stained by DAPI (blue), Scale bar, $200 \mu \mathrm{m}$. B. CD90 (green) immunofluorescent staining of cardiac fibroblasts, nucleus was stained by DAPI (blue), Scale bar, $100 \mu \mathrm{m}$. C. CD 90 positive cardiac fibroblasts were isolated by flow cytometry. Images of cultured CD 90 positive cells were presented by light microscope, scale bar, $200 \mu \mathrm{m}$. FSP1, fibroblasts specific protein 1. 

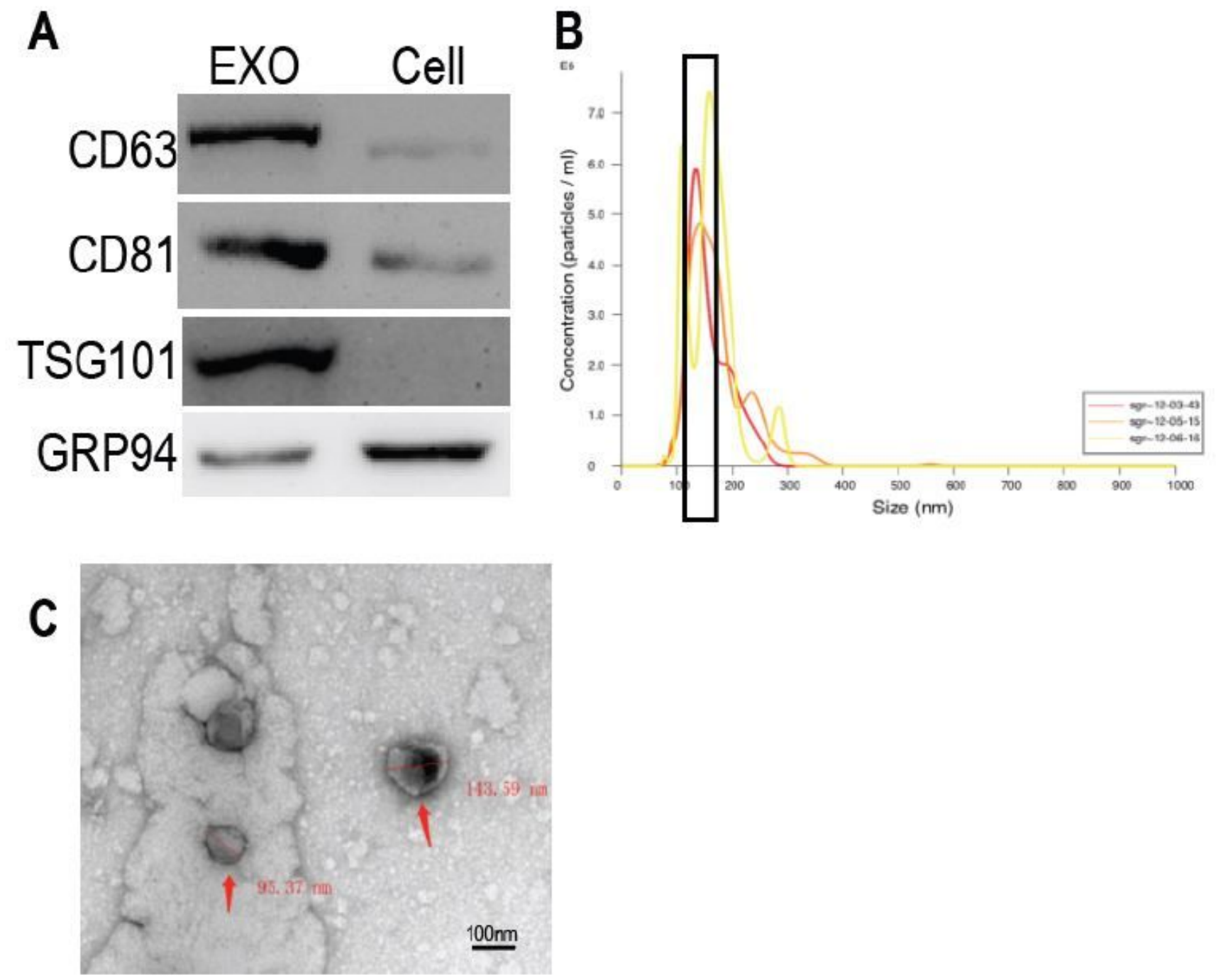

\section{Figure 2}

Characterization of exosome derived from CD90 positive cardiac fibroblasts exosome. A. Assessment of CD63, CD81, TSG101 and GRP94 expression in exosome by western blot. B. Size distribution of particles among exosomes using NanoSight imaging. C. Electron microscopy images of exosome. Scale bar, $100 \mathrm{~nm}$. 
A

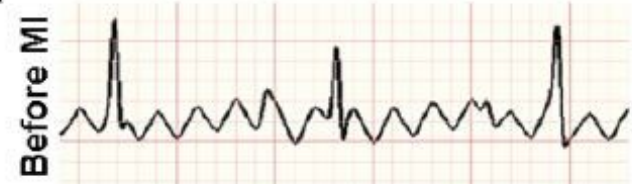

B
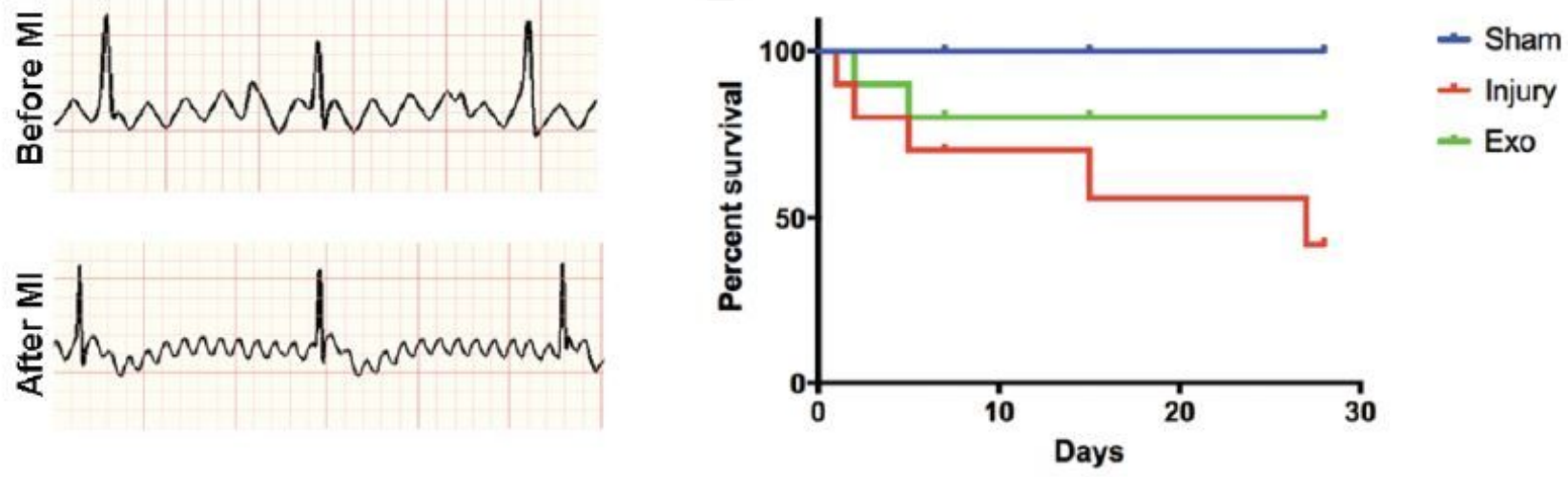

C

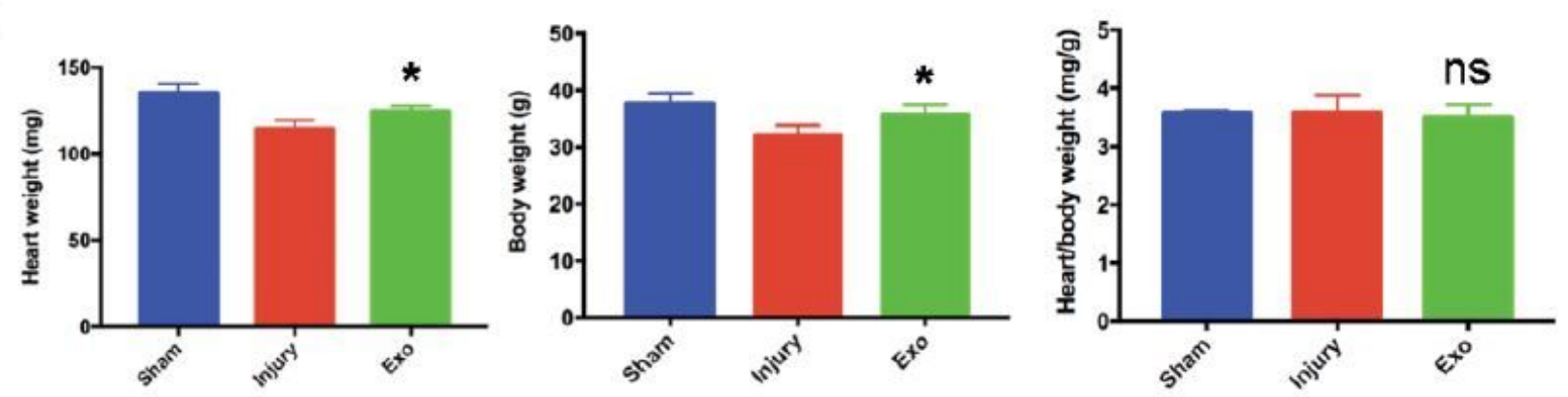

Figure 3

Comparisons of survival, heart weight and body weight. A. Electrography image of heart before myocardial infarction and after myocardial infarction. B. Survival curves of mice after injury. C. Analysis of heart weight, body weight and Heart/body weight ratio. Data are shown as mean $\pm S E M, *<0.05$, ns $>0.05$ compared with injury groups. 
A

Sham

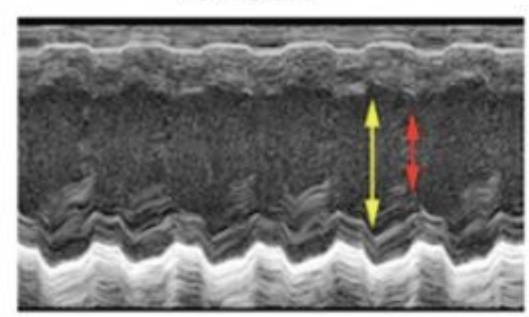

B
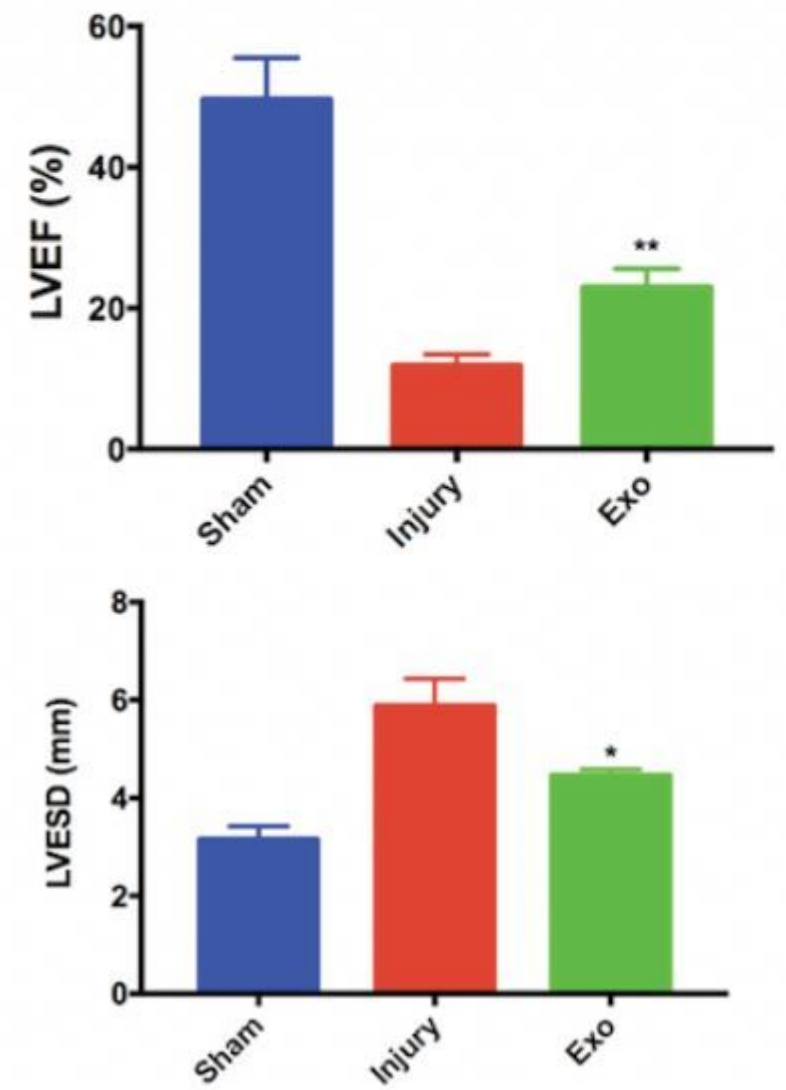

Injury

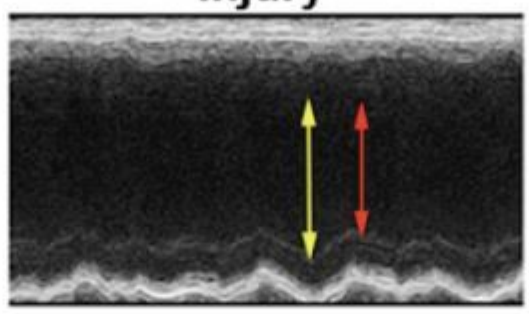

Exo
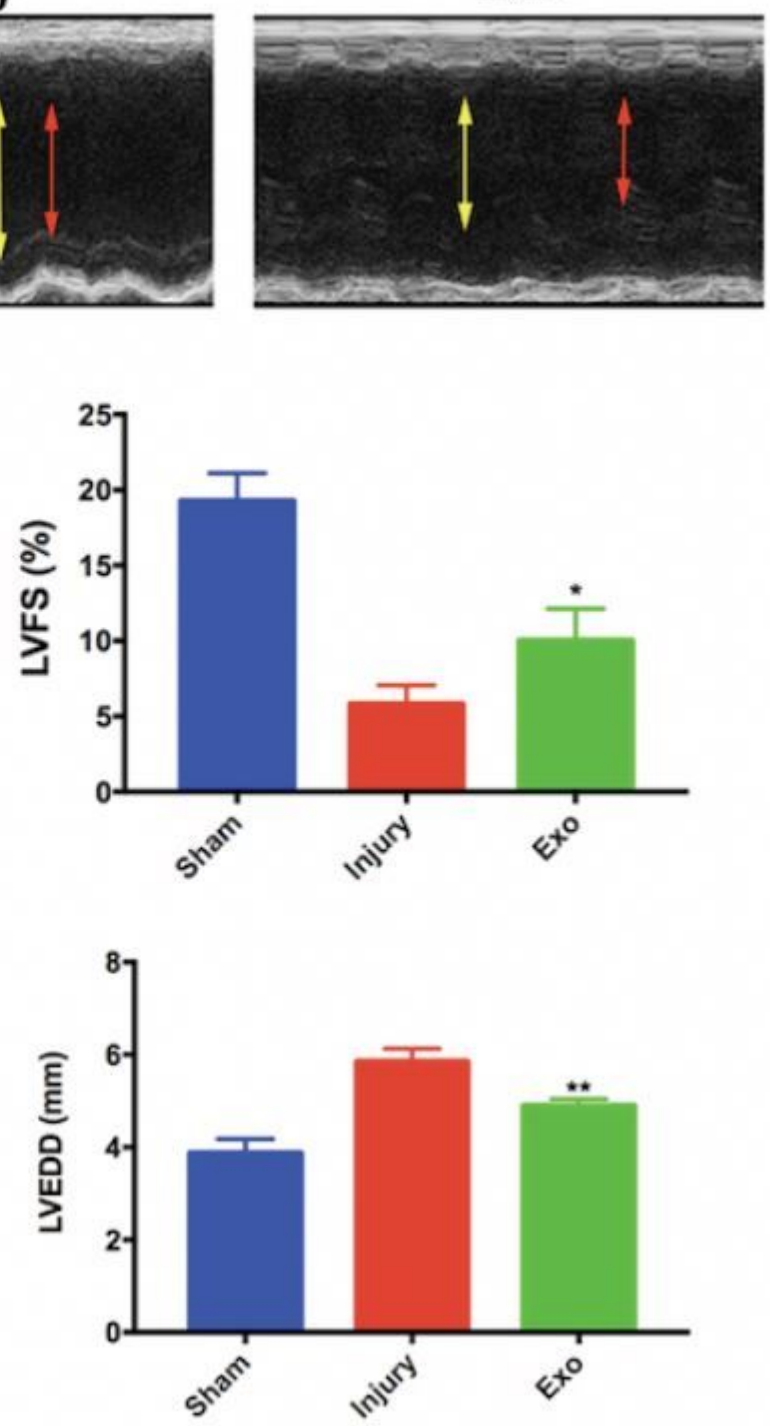

Figure 4

The effects of exosome on heart function after injury. Heart functions were analyzed at 4 weeks after injury. A. ultrasonic echocardiography images of hearts, yellow line, end of diastole, red line, end of systole. B. Comparison of LVEF, LVFS, LVESD, LVEDD in each group by echocardiography. Data are shown as mean $\pm S E M, \otimes p<0.05, \star \star p<0.01$ compared to injury groups. 


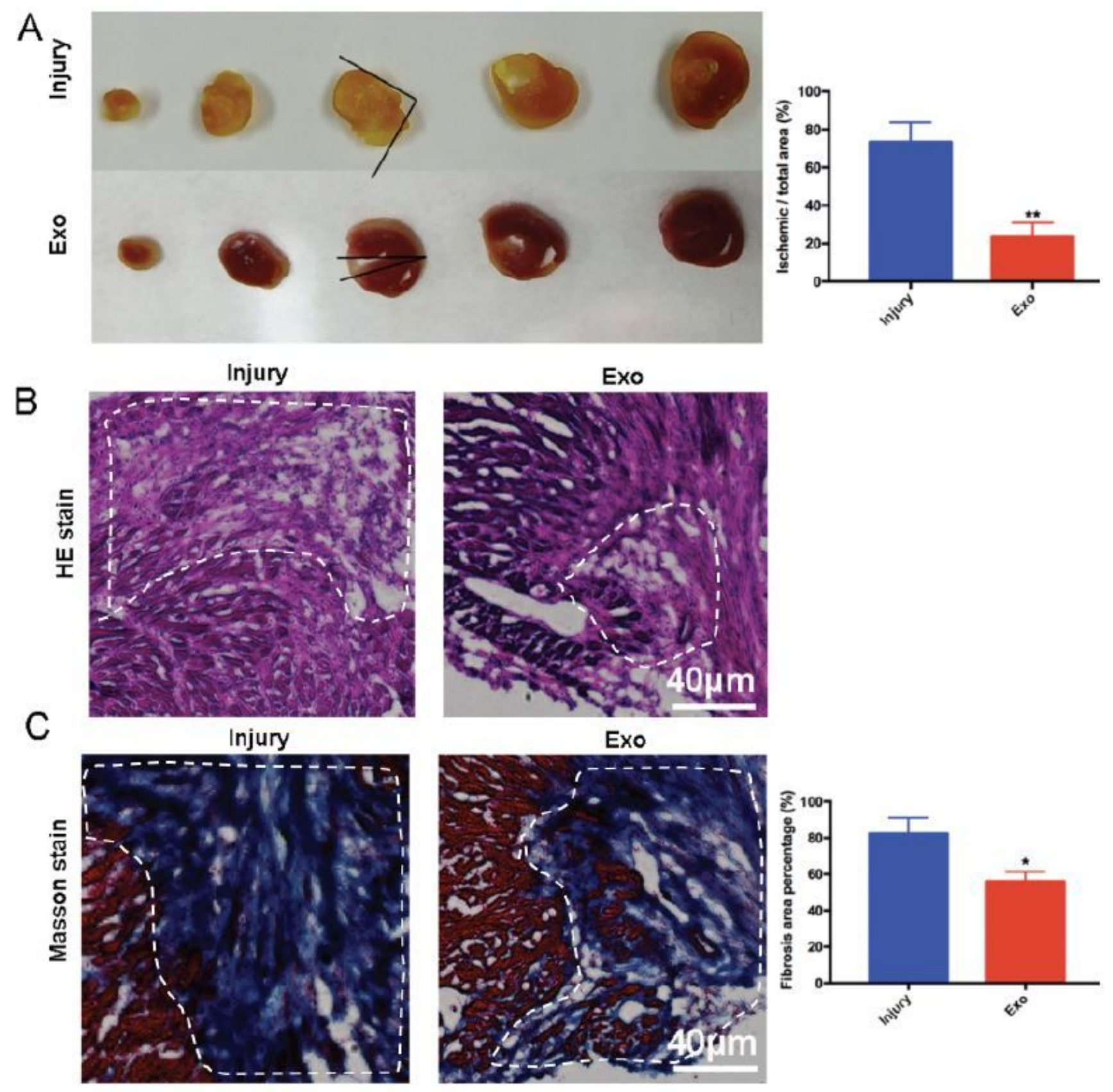

Figure 5

The pathological regulatory effects of exosome on heart after myocardial infarction. Hearts were collected at 4 weeks after surgery. A. The presentative images of TTC stain in hearts, viable heart muscles were red-stained, infarction tissues were pale, ischemic ratio was presented as a percentage of total area of left ventricular using the middle heart section. B. Images of hearts by HE stains, scale bar, $40 \mu \mathrm{m}$. C. cardiac fibrosis was represented by Masson stain, the fibrosis area ratio was determined by a percentage of area per the high-power field, scale bar, $40 \mu \mathrm{m}$. Data are shown as mean $\pm S E M, \otimes p<0.05, \star \star p<0.01$ compared to injury groups. 

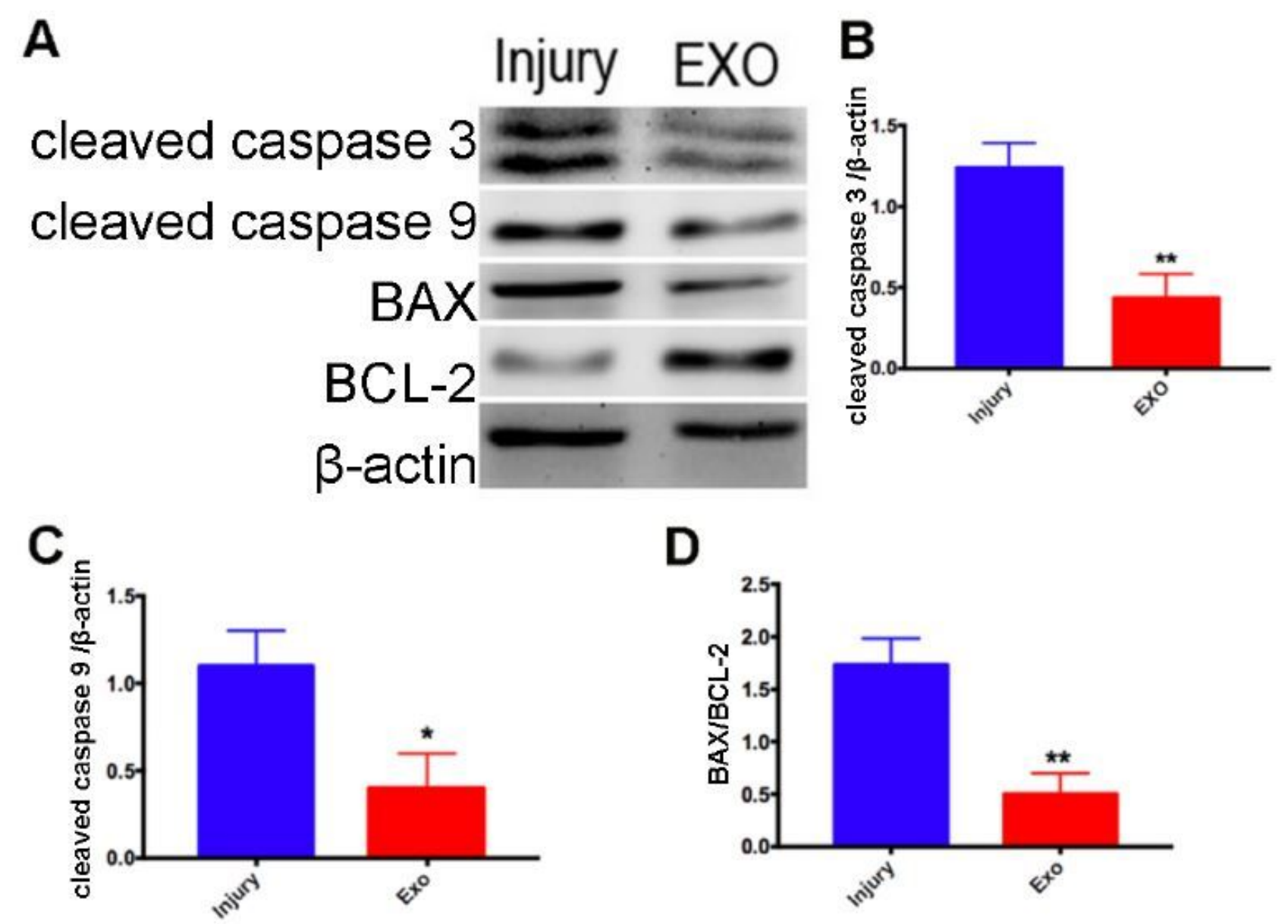

D

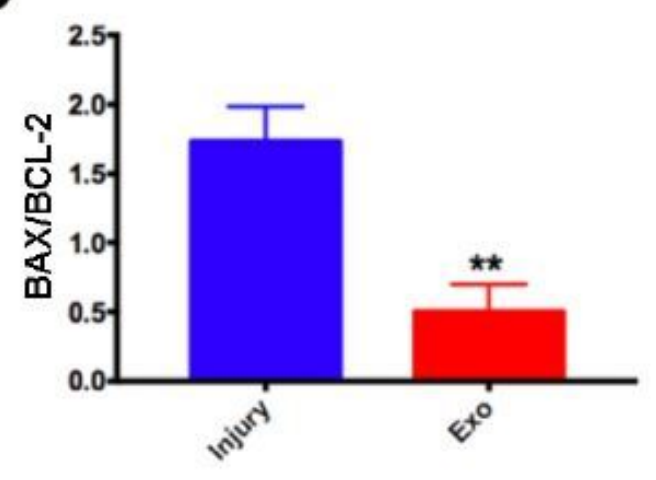

Figure 6

The apoptotic effects of exosome on heart after myocardial infarction. Heart tissues were collected at day 3 post injury. A. Representative western blot images of cleaved caspase 3, cleaved caspase 9, BAX BCL-2 and BETA-ACTIN from heart tissues. B-D. Comparison of cleaved caspase 3, cleaved caspase 9

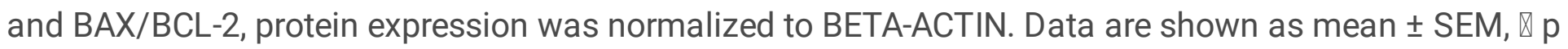
$<0.05, * \star p<0.01$ compared to injury groups. 


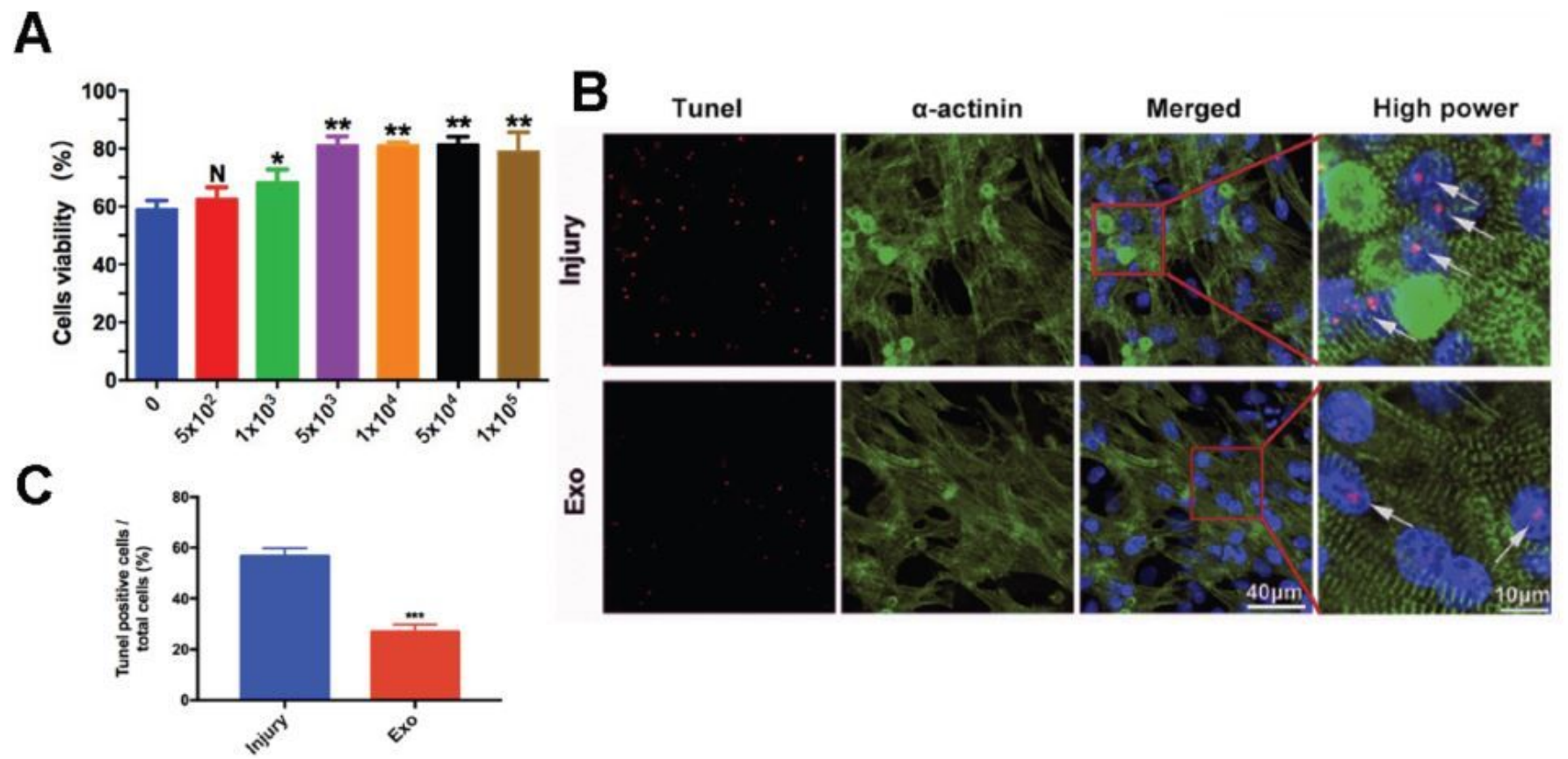

\section{Figure 7}

The effects of exosome on cardiomyocytes after hypoxia in vitro. A. MTT viability data of cardiomyocytes under hypoxia condition with or without exosome coincubation, Absorbance data were analyzed via one-way ANOVA and posthoc Tukey's test. Data are shown as mean \pm SEM (ns $p>0.05, \nabla p<$ 0.05 , $* * p<0.01$ compared to exosome-free co-incubation). B. Apoptosis detection in cardiomyocytes under hypoxia condition by immunofluorescence. red, Tunel; green a-actinin, blue, DAPI. C. Comparison of a percentage of tunel positive cells. Data are shown as mean \pm SEM, $* \star \star p<0.001$ compared to injury groups. 


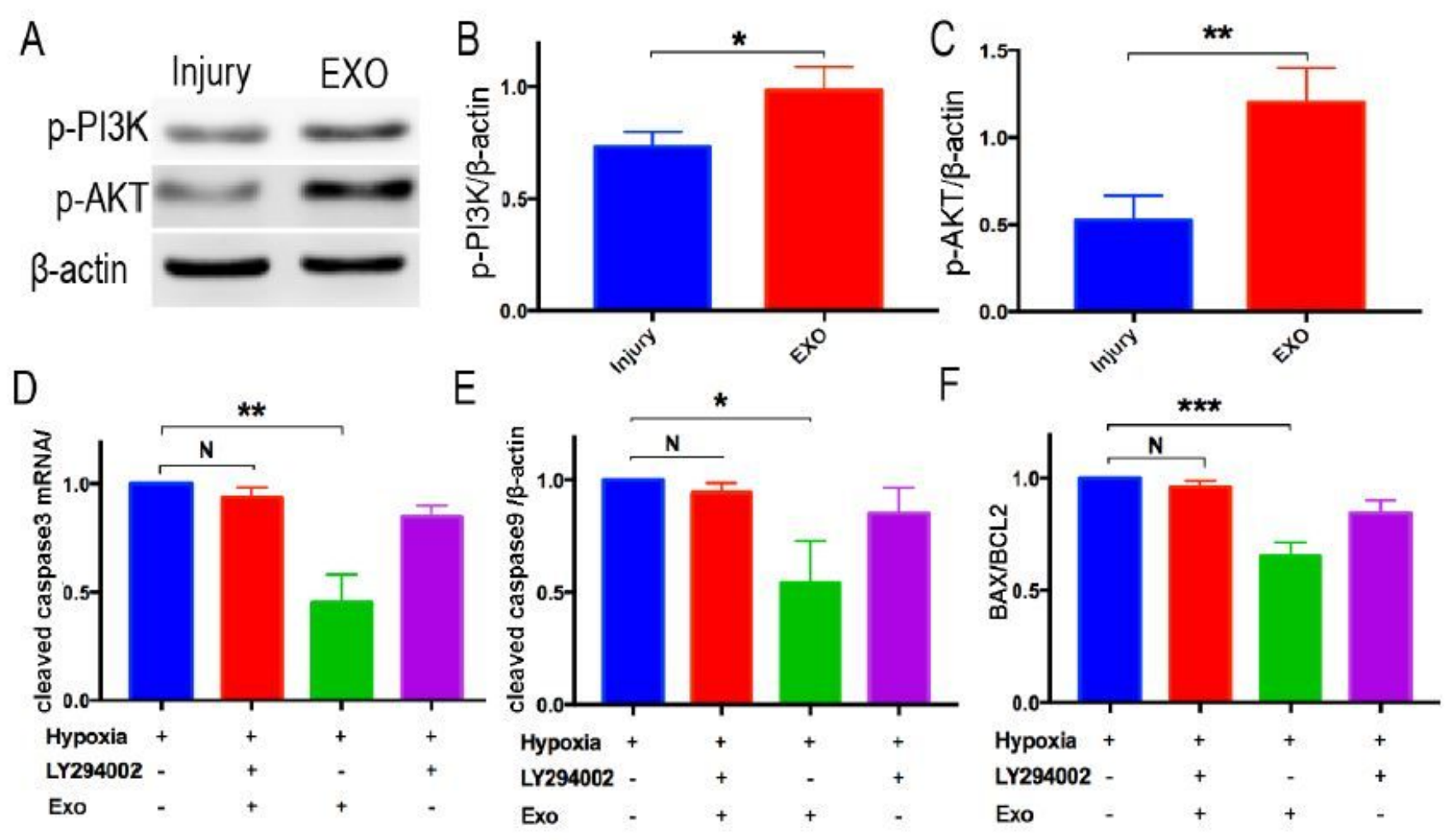

\section{Figure 8}

Exosome co-incubation decreased apoptosis of myocardiocytes induced by hypoxia via PI3K/AKT signaling pathway. A. Assessment of p-PI3K, p-AKT and BETA-ACTIN expression in cardiomyocytes in vitro by western blot. B-C. Analyze of p-PI3K and p-AKT expression, and protein expression was

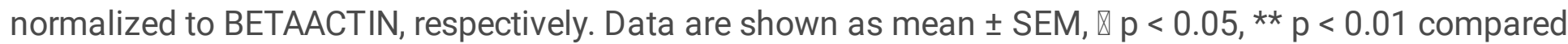
to injury groups. D-F. Relative mRNA expression of cleaved caspase 3, cleaved caspase 9 and BAX/BCL-2 in cardiomyocytes under hypoxia condition with or without supplement of LY294002, gene expression was normalized to BETA-ACTIN. Data are shown as mean \pm SEM, ns $p>0.05, \otimes p<0.05, * \star p<0.01$. 